\title{
NAKAYAMA AUTOMORPHISMS OF TWISTED TENSOR PRODUCTS
}

\author{
Y. SHEN, G.-S. ZHOU, AND D.-M. LU
}

\begin{abstract}
AвstRact. In this paper, we study homological properties of twisted tensor products of connected graded algebras. We focus on the Ext-algebras of twisted tensor products with a certain form of twisting maps firstly. We show those Ext-algebras are also twisted tensor products, and depict the twisting maps for such Ext-algebras indepth. With those preparations, we describe Nakayama automorphisms of twisted tensor products of noetherian Artin-Schelter regular algebras.
\end{abstract}

\section{INTRODUCTION}

The appearance of twisted tensor product of two algebras provides an impactful solution to the questions about "factorization" and "product" in quantum groups and noncommutative geometry ([3, 4, 15]). Compared with the common tensor product, twisted tensor product makes up for the limitation of too much commutativity, and is a suitable choice for constructing larger classes of noncommutative algebras. Roughly speaking, twisted tensor product $A \otimes^{\tau} B$ of algebras $A$ and $B$ is an associative algebra, which is $A \otimes B$ as vector spaces with the multiplication defined by a twisting map $\tau: B \otimes A \rightarrow A \otimes B$. The notion also has been called $R$-smash product in several recent papers $([3,12,18])$.

For our research interests, $A$ and $B$ are always finitely generated algebras. In order to obtain nice combinatorial and homological properties, it is reasonable to demand the twisting map $\tau$ being determined by the action on generating set of $B \otimes A$. This requirement can be realized by an algebra homomorphism $\sigma=\left(\sigma_{i j}\right)$ from $A$ to matrix algebra $\mathbb{M}_{m}(A)$ and a $\sigma$-derivation $\delta=\left(\delta_{1}, \cdots, \delta_{m}\right)^{T}$ from $A$ to $A^{\oplus m}$, where $m$ is the number of elements in the minimal generating set of $B$. In this case, we write $\tau=(\sigma, \delta)$. Significantly, classical Ore extensions and double Ore extensions with zero tails (see [20] for detail) are both special cases of such twisted tensor products.

Artin-Schelter regular (AS-regular, for short) algebras play an important role in noncommutative algebras ([1]). The classification and construction of AS-regular algebras have acquired plentiful achievements. Ore extensions and double Ore extensions are commonly used to construct AS-regular algebras. Wang and the first and third named authors lead twisted tensor products into construction of AS-regular algebras in [18]. The result tells that the twisted tensor product of AS-regular algebras $A$ and $B$ preserves AS-regularity in case $\sigma$ is invertible (see Definition 1.8) and $B$ has a pure resolution, namely, each term of the minimal free resolution of the trivial module concentrates in one degree ([18, Theorem 0.3]). The typical examples of algebras with pure resolutions include $(d$-)Koszul algebras and piecewise Koszul algebras. The AS-regularity of twisted tensor products inspires us to study more homological properties of this construction.

Nakayama automorphism is one of important homological invariants for AS-regular algebras, equivalently connected graded skew Calabi-Yau algebras. From the view of skew Calabi-Yau categories (see [11]),

2010 Mathematics Subject Classification. 16E65, 16W50, 14A22.

Key words and phrases. Artin-Schelter regular algebras, twisted tensor products, Ext-algebras, Nakayama automorphisms. 
the Nakayama automorphisms correspond to Serre functors of categories. In other side, Nakayama automorphisms control Hopf actions ([5, 9]). As special cases, Nakayama automorphisms of Ore extensions and trimmed double Ore extensions of Koszul AS-regular algebras have been studied in [7, 21], respectively. Our aim is to give a description of the Nakayama automorphism of twisted tensor product $A \otimes^{\tau} B$ of noetherian AS-regular algebras $A$ and $B$, where the graded twisting map $\tau=(\sigma, \delta): B \otimes A \rightarrow A \otimes B$. It is the initial purpose of this paper.

To achieve the goal, we start from the study of Ext-algebras of twisted tensor products. The Ext-algebra $E(A):=\underline{\operatorname{Ext}}_{A}^{*}(k, k)$ is an incredibly powerful tool used in many areas of mathematics for a connected graded algebra $A$. We focus on the Yoneda product of it. Let $A$ and $B$ be two connected graded algebras with $B$ having a pure resolution, and let $A \otimes^{\tau} B$ be a twisted tensor product for some graded twisting map $\tau=(\sigma, 0)$. We construct a minimal free resolution of trivial module of $A \otimes^{\tau} B$ (see Proposition 2.10), by the complex obtained in [18, Theorem 0.2]. This free resolution helps us bridge the relationships among the Ext-algebras $E(A), E(B)$ and $E\left(A \otimes^{\tau} B\right)$. As a generalization of [12, Theorem 3.7], we get a result about Ext-algebras of twisted tensor products.

Theorem A. Let $A$ and $B$ be two connected Ext-finite graded algebras. Let $\tau=(\sigma, 0): B \otimes A \rightarrow A \otimes B$ be a graded linear map such that $A \otimes^{\tau} B$ is a twisted tensor product. Denote the Ext-algebras of $A, B$ and $A \otimes^{\tau} B$ by $E(A), E(B)$ and $E\left(A \otimes^{\tau} B\right)$, respectively. Suppose $B$ has a pure resolution, then as bigraded algebras

$$
E\left(A \otimes^{\tau} B\right) \cong E(B) \otimes^{\tau_{E}} E(A),
$$

for some bigraded linear map $\tau_{E}: E(A) \otimes E(B) \rightarrow E(B) \otimes E(A)$.

From the theorem above, we know the Yoneda product of Ext-algebra $E\left(A \otimes^{\tau} B\right)$ depends on the bigraded linear map $\tau_{E}$, which is relevant to the graded twisting map $\tau=(\sigma, 0)$. So the properties of graded linear map $\sigma$ from $A$ to a matrix algebra $\mathbb{M}_{m}(A)$ over $A$, where $m$ is the number of elements in the minimal generating set of $B$, are determining factors for the Yoneda product of Ext-algebra $E\left(A \otimes^{\tau} B\right)$.

When we restrict ourselves on AS-regular algebras, there are two important homological invariants arising from $\sigma$. The first one is homological determinant hdet $\sigma$, that is an $m \times m$ matrix over based field $k$ coming from the linear transformation of the direct sum $E(A)^{\oplus m}$ induced by $\sigma$. It is a generalization for homological determinants of algebra automorphisms defined in [6].

Besides, there is an automorphism $\operatorname{det} \sigma$ of $A$ such that as $(A, A)$-bimodules

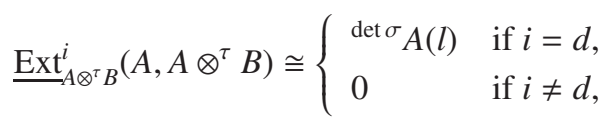

for some integers $d, l$. The automorphism $\operatorname{det} \sigma$ is called determinant of $\sigma$. It is a generalization of determinant defined for double Ore extensions in [20]. If $B$ is a polynomial algebra, $\operatorname{det} \sigma$ coincides with the classical determinant of matrices (see Example 2.15). Such two invariants of $\sigma$ make us describe the Yoneda product of Ext-algebra $E\left(A \otimes^{\tau} B\right)$ further, and provide an approach to study Nakayama automorphisms of twisted tensor products.

By using the theory of $A_{\infty}$-algebras, we have known an algebra is AS-regular if and only if its Extalgebra is a Frobenius algebra (see [8]). Frobenius algebras have classical Nakayama automorphisms. By a result of Reyes, Rogalski and Zhang, Nakayama automorphisms of noetherian AS-regular algebras are dual to classical Nakayama automorphisms of their Ext-algebras (see [11]). Our question turns to compute the Nakayama automorphisms of Ext-algebras. 
Let $A \otimes^{\tau} B$ be a twisted tensor product of two connected graded algebras $A, B$, where the graded twisting map $\tau=(\sigma, \delta): B \otimes A \rightarrow A \otimes B$ with nonzero $\sigma$-derivation. Note that the correlative graded linear map $\bar{\tau}=(\sigma, 0): B \otimes A \rightarrow A \otimes B$ is also a graded twisting map, that is, $A \otimes \otimes^{\bar{\tau}} B$ is a twisted tensor product. The observation about the Yoneda product from the homological determinants and determinants mentioned above helps us describe the bigraded linear map $\tau_{E}$ for $A \otimes^{\bar{\tau}} B$ occurred in Theorem $\mathrm{A}$. So we can compute the Nakayama automorphism of Ext-algebra $E\left(A \otimes^{\bar{\tau}} B\right)$. In particular, $A \otimes^{\bar{\tau}} B$ is an associated graded algebra of $A \otimes^{\tau} B$ for a canonical filtration. Following the properties of filtered algebras, we realize our ultimate goal.

Theorem B. Let $A$ and $B$ be both noetherian AS-regular algebras generated in degree 1 , and $Y=\left\{y_{i}\right\}_{i=1}^{m}$ be a minimal generating set of $B$. Let $\tau=(\sigma, \delta): B \otimes A \rightarrow A \otimes B$ be a graded twisting map such that associated twisted tensor product $A \otimes^{\bar{\tau}} B$ is noetherian. Denote the Nakayama automorphisms of $A, B$ and $A \otimes^{\tau} B$ by $\mu_{A}, \mu_{B}$ and $\mu_{A \otimes^{\tau} B}$, respectively. Suppose $B$ has a pure resolution and $\sigma$ is invertible, then Nakayama automorphism $\mu_{A \otimes^{\top} B}$ satisfies

$$
\begin{aligned}
& \mu_{A \otimes^{\tau} B \mid A}=(\operatorname{det} \sigma)^{-1} \circ \mu_{A}, \\
& \mu_{A \otimes^{\tau} B}\left(\begin{array}{c}
1 \otimes^{\tau} y_{1} \\
1 \otimes^{\tau} y_{2} \\
\vdots \\
1 \otimes^{\tau} y_{m}
\end{array}\right)=\operatorname{hdet} \sigma \cdot\left(A \otimes^{\tau} \mu_{B}\right)\left(\begin{array}{c}
1 \otimes^{\tau} y_{1} \\
1 \otimes^{\tau} y_{2} \\
\vdots \\
1 \otimes^{\tau} y_{m}
\end{array}\right)+\left(\begin{array}{c}
a_{1} \otimes^{\tau} 1 \\
a_{2} \otimes^{\tau} 1 \\
\vdots \\
a_{m} \otimes^{\tau} 1
\end{array}\right),
\end{aligned}
$$

where $a_{1}, a_{2}, \cdots, a_{m} \in A$ and $\left(A \otimes^{\tau} \mu_{B}\right)\left(1 \otimes^{\tau} y_{i}\right)=1 \otimes^{\tau} \mu_{B}\left(y_{i}\right)$ for $i=1, \cdots, m$.

This theorem coincides with the results obtained in [7, Theorem 2] and [21, Theorem 2] for the case of Ore extensions and trimmed double Ore extensions of Koszul AS-regular algebras.

The paper is organized as follows. In Section 1, we fix notations and recall some definitions and results, as well as the definition of homological determinant is given. In Section 2, we review some basic results of twisted tensor products, and discuss homological properties of twisted tensor products, including a minimal free resolution of trivial module of twisted tensor product, and the determinant for a graded algebra homomorphism from a connected graded algebra to a matrix algebra over it. In Section 3 , we focus on the Yoneda product of Ext-algebras, and Theorem $\mathrm{A}$ is proved. In Section 4 we prove Theorem B In Section 5 we give some applications of Theorem B.

Throughout the paper, $k$ is a fixed field. All vector spaces and algebras are over $k$. Unless otherwise stated, tensor product $\otimes$ means $\otimes_{k}$.

\section{Preliminaries}

1.1. Notations and Definitions. In this section, we fix basic notations and recall definitions for this paper.

Let $A=\bigoplus_{i \in \mathbb{Z}} A_{i}$ be a graded algebra. If $\operatorname{dim} A_{i}<\infty$ for all $i, A$ is called locally finite. A locally finite graded algebra $A$ is called connected, if $A_{i}=0$ for all $i<0$ and $A_{0}=k$. In this case, we write $\varepsilon_{A}: A \rightarrow k$ to be the augmentation for $A$.

We denote by Gr $A$ the category of left graded $A$-modules with morphisms consisting of left $A$-module homomorphisms preserving degrees. We identify $\operatorname{Gr} A^{o}$ (resp. Gr $A^{e}$ ) with the category of graded right 
$A$-modules (resp. graded $A$-bimodules), where $A^{o}$ is the opposite algebra of $A$ and $A^{e}=A \otimes A^{o}$ is the enveloping algebra of $A$. Unless otherwise stated, all modules are left modules in this paper.

For each integer $i$, we define $i$-th shift functor $-(i):$ Gr $A \rightarrow$ Gr $A$ by sending a graded module $M$ to $M(i)$, where $M(i)$ is a graded $A$-module with new grading $M(i)_{j}=M_{i+j}$ for each $j \in \mathbb{Z}$, and acting on morphisms as the identity map. Let $M, N \in \operatorname{Gr} A$ and $i$ be an integer, write graded vector spaces

$$
\underline{\operatorname{Hom}}_{A}(M, N)=\bigoplus_{j \in \mathbb{Z}} \operatorname{Hom}_{\mathrm{Gr} A}(M, N(j)) \text { and } \quad \underline{\operatorname{Ext}}_{A}^{i}(M, N)=\bigoplus_{j \in \mathbb{Z}} \operatorname{Ext}_{\mathrm{Gr} A}^{i}(M, N(j)) .
$$

Note that $\underline{\operatorname{Hom}}_{A}(M, N)$ and $\underline{\operatorname{Ext}}_{A}^{i}(M, N)$ become graded left modules if $M$ is a graded bimodule, graded right modules if $N$ is a graded bimodule, and graded bimodules if $M$ and $N$ are both graded bimodules. Similar remarks apply to the tensor product $L \otimes_{A} M$ of a graded right $A$-module $L$ ( $L \otimes_{A} M$ is graded by $\operatorname{deg}(l \otimes m)=\operatorname{deg} l+\operatorname{deg} m$ when $l \in L$ and $m \in M$ are homogeneous).

Let $M$ be a graded $A$-bimodule, and let $\mu, v$ be graded algebra automorphisms of $A$. Then ${ }^{\mu} M^{v}$ denotes the twisted $A$-bimodule such that ${ }^{\mu} M^{v}=M$ as abelian groups, and where $a * m * a^{\prime}=\mu(a) m v\left(a^{\prime}\right)$ for all $a, a^{\prime} \in A$ and $m \in M$. If $\mu=$ id (resp. $v=$ id), we use $M^{v}$ (resp. ${ }^{\mu} M$ ) for ${ }^{\text {id }} M^{v}$ (resp. ${ }^{\mu} M^{\text {id }}$ ). Note that if $A$ is connected, it follows that $A^{\mu} \cong A^{v}$ as graded $A$-bimodules if and only if $\mu=v$.

Definition 1.1. A connected graded algebra A is called an Artin-Schelter regular (AS-regular, for short) algebra of type $(d, l)$, if the following conditions hold:

(AS1) $A$ has finite global dimension $d$.

(AS2) $\underline{\operatorname{Ext}}_{A}^{d}(k, A)=k(l)$ and $\underline{\operatorname{Ext}}_{A}^{i}(k, A)=0$ for $i \neq d$.

If $A$ is an AS-regular algebra of type $(d, l)$, then by [19, Proposition 4.5 (b)], there exists a unique graded automorphism $\mu$ of $A$ such that

$$
\underline{\operatorname{Ext}}_{A^{e}}^{i}\left(A, A^{e}\right) \cong\left\{\begin{array}{ll}
0 & i \neq d, \\
A^{\mu}(l) & i=d,
\end{array} \quad \text { as graded } A\right. \text {-bimodules. }
$$

In this case, the automorphism $\mu$ is called the Nakayama automorphism of $A$, and always denoted by $\mu_{A}$.

We denote by $\mathrm{Ch}(\mathrm{Gr} A)$ the category of cochain complexes of graded left $A$-modules with cochain complex morphisms. For each integer $i$, the $i$-th shift of cochain complex is a functor $-[i]: \operatorname{Ch}(\operatorname{Gr} A) \rightarrow$ $\operatorname{Ch}(\operatorname{Gr} A)$ sending $X \cdot$ to a new cochain complex $X[i]$ with terms $X[i]^{j}=X^{j+i}$ and differential $(-1)^{i} d_{X}$, and acting on morphisms as the identity map. For any $\left(X^{\cdot}, d_{X}\right) \in \mathrm{Ch}(\mathrm{Gr} A)$ and $\left(Y^{\cdot}, d_{Y}\right) \in \mathrm{Ch}\left(\operatorname{Gr} A^{o}\right), Y^{\cdot} \otimes_{A} X^{\cdot}$ is a cochain complex where $n$-th term is $\bigoplus_{i+j=n} Y^{i} \otimes_{A} X^{j}$ and differential $d\left(y \otimes_{A} x\right)=d_{Y}(y) \otimes_{A} x+(-1)^{i} y \otimes d_{X}(x)$ for $x \in X^{j}$ and $y \in Y^{i}$.

When dealing with bigraded cases, we follow the Koszul sign rule for the first degree (such as homological degree) throughout the paper: when interchanging bihomogeneous elements of degree $\left(i_{1}, j_{1}\right)$ and $\left(i_{2}, j_{2}\right)$, multiply by $(-1)^{i_{1} i_{2}}$, which ignores the second degree. For example, a cochain complex $X^{\cdot}$ in $\mathrm{Ch}(\mathrm{Gr} A)$ can be considered as a bigraded vector spaces $\bigoplus_{i} X^{i}$ with differential $d_{X}$ of degree $(1,0)$. Following the Koszul sign rule, the differential of $Y \cdot \otimes_{A} X^{\cdot}$ has the form of $d_{Y} \otimes_{A} \operatorname{id}_{X}+\operatorname{id}_{Y} \otimes_{A} d_{X}$.

1.2. Ext-algebras. Let $A$ be a connected graded algebra. There exists a graded free resolution $P \cdot{ }^{*}{ }_{A} k$

$$
P^{\cdot}=\left(\cdots \rightarrow A \otimes V_{3} \stackrel{d_{P}^{-3}}{\longrightarrow} A \otimes V_{2} \stackrel{d_{P}^{-2}}{\longrightarrow} A \otimes V_{1} \stackrel{d_{P}^{-1}}{\longrightarrow} A \rightarrow 0 \rightarrow \cdots\right) \stackrel{\varepsilon_{A}}{\longrightarrow} \quad{ }_{A} k,
$$


where $V_{i}$ is a graded vector space, such that $\operatorname{Im} d_{P}^{-i} \subseteq A_{\geq 1} \otimes V_{i-1}$ for all $i=1,2, \cdots$. Here, we let $V_{0}=k$ and identify $A$ with $A \otimes k$. Such a graded free resolution is unique up to isomorphism in $\operatorname{Ch}(\operatorname{Gr} A)$, and is called the minimal free resolution of ${ }_{A} k$.

Here and from now on, $(-)^{*}$ denotes the graded dual functor of graded vector spaces. It is easy to see that the complex $\underline{\operatorname{Hom}}_{A}\left(P^{\cdot}, k\right)$ has zero differential. Hence the graded vector space $\underline{\operatorname{Ext}}_{A}^{i}(k, k)=\underline{\operatorname{Hom}}_{A}(A \otimes$ $\left.V_{i}, k\right) \cong V_{i}^{*}$ for all $i \geq 0$. We say $A$ is Ext-finite if all $\operatorname{Ext}_{A}^{i}(k, k)$ are of finite dimension, or equivalently, all $V_{i}$ are finite dimensional, such as AS-regular algebras ([14, Proposition 3.1]). Also, we say $A$ has a pure resolution if each $V_{i}$ concentrates in one degree. The examples of algebras with a pure resolution contain $(d$-)Koszul algebras and piecewise Koszul algebras.

The Ext-algebra of $A$ is a bigraded vector space

$$
E(A):=\bigoplus_{(i, j) \in \mathbb{Z}^{2}} \underline{\operatorname{Ext}}_{A}^{i}(k, k)_{j}
$$

equipped with the Yoneda product, where the second degree comes from the grading of $A$, written as subscript. The $(i, j)$-bihomogeneous piece of $E(A)$ is $E^{i}(A)_{j}=\operatorname{Hom}_{\mathrm{Gr} A}\left(P^{-i}, k(j)\right)$.

The paper [12] shows Ext-algebras for connected graded algebras give rise to a functor. We review it here for completion. Let $A, B$ be two connected graded algebras, and $f: A \rightarrow B$ be a graded algebra homomorphism. We define a bigraded algebra homomorphism $E(f): E(B) \rightarrow E(A)$ as follows.

There is a morphism of cochain complexes $\widetilde{f}: P^{\cdot} \rightarrow Q \cdot$ in $\mathrm{Ch}(\mathrm{Gr} A)$ lifting $f$, such that $\varepsilon_{B} \circ f=\varepsilon_{A}$, where $P \cdot Q \cdot$ are minimal free resolutions of ${ }_{A} k,{ }_{B} k$ respectively. Note that the graded algebra homomorphism $f$ makes all $B$-modules endow with $A$-module structures, then

$$
E(f): E(B) \cong \underline{\operatorname{Hom}}_{B}\left(Q^{\prime}, k\right) \hookrightarrow \underline{\operatorname{Hom}}_{A}\left(Q^{\prime}, k\right) \stackrel{\left.\operatorname{Hom}_{A} \widetilde{f}, k\right)}{\longrightarrow} \underline{\operatorname{Hom}}_{A}\left(P^{\cdot}, k\right) \cong E(A) .
$$

It can be checked $E(f)$ is an algebra homomorphism straightforwardly.

Lemma 1.2. [12, Theorem 2.4] Taking Ext-algebra E(-) is a contravariant functor from the category of connected graded algebras to the category of bigraded algebras.

Definition 1.3. Let $E=\bigoplus_{(i, j) \in \mathbb{Z}^{2}} E_{j}^{i}$ be a finite dimensional bigraded algebra with $E_{0}^{0}=k$. We say $E$ is a Frobenius algebra of type $(-d, l)$, if there is a nondegenerate associative bigraded bilinear form $\langle-,-\rangle: E \otimes E \rightarrow k$ of degree $(-d, l)$. By the nondegeneracy of the bilinear form, there exists a bigraded automorphism $\mu$ of $E$ such that

$$
\langle a, b\rangle=(-1)^{i_{1} i_{2}}\langle\mu(b), a\rangle,
$$

for $a \in E^{i_{1}}, b \in E^{i_{2}}$. It implies $E^{*} \cong{ }^{\mu} E(l)$ as bigraded $E^{e}$-modules. The unique automorphism $\mu$ is called the (classical) Nakayama automorphism, denoted by $\mu_{E}$. For more detail, see [13].

The Ext-algebra $E(A)$ may be considered as a "dual" of $A$ in some sense, and recover lots of properties of $A$. We have following important results for AS-regularity and Nakayama automorphisms.

Theorem 1.4. Let $A$ be a connected graded algebra, and $E(A)$ be the Ext-algebra of $A$.

(a) [8, Corollary D] The algebra A is AS-regular of type $(d, l)$ if and only if $E(A)$ is a Frobenius algebra of type $(-d, l)$. 
(b) [11, Theorem 4.2] If A is noetherian AS-regular of type $(d, l)$ generated by degree 1 . Let $\mu_{A}$ and $\mu_{E}$ be Nakayama automorphisms of $A$ and $E(A)$, respectively. Then

$$
\mu_{E \mid E^{1}(A)}=\left(\mu_{A_{\mid A_{1}}}\right)^{*},
$$

where $E^{1}(A)$ is identified with $A_{1}^{*}$.

Remark 1.5. The signs in the bigraded bilinear forms of Frobenius algebras come from the Koszul sign rule, which is different to the one defined in [11]. So there is no additional sign in the formula for Nakayama automorphisms in Theorem 1.4 b), by a modification of signs to the proof of [11, Theorem 4.2].

1.3. Homological determinant. Let $A$ be a connected graded algebra and $m$ be an arbitrary positive integer. Graded algebra homomorphisms from $A$ to matrix algebra $\mathbb{M}_{m}(A)$ play a key role in the discussion of twisted tensor products in the rest of paper. In this subsection, we introduce a definition of homological determinant for such graded algebra homomorphisms, which is a generalization of homological determinant defined in [6, 21]. Before that, we need some preparations.

Definition 1.6. Let $M$ be a graded $A$-module and $U \cong k(l)^{\oplus m}$ be a graded vector space for some integer $l$. Let $\phi=\left(\phi_{i j}\right): A \rightarrow \mathbb{M}_{m}(A)$ be a graded algebra homomorphism, where each $\phi_{i j}: A \rightarrow A$ is a morphism of graded vector spaces. The $\phi$-twisted graded A-module structure on $M \otimes U$ is given by

$$
a *\left(\sum_{i=1}^{m} x_{i} \otimes e_{i}\right)=\sum_{j=1}^{m}\left(\sum_{i=1}^{m} \phi_{j i}(a) x_{i}\right) \otimes e_{j},
$$

where $a \in A, x_{i} \in M$ and $\left\{e_{i}\right\}_{i=1}^{m}$ is the canonical coordinate basis of $U$. Such a graded $A$-module is denoted by ${ }^{\phi}(M \otimes U)$. In particular, it is the usual twisted module structure when $m=1$.

Similarly, we can define $\phi$-twisted graded right $A$-modules.

Remark 1.7. In fact, we can understand the $\phi$-twisted graded $A$-module structure by a multiplication of matrices. Note that $M \otimes U \cong M(l)^{\oplus m}$, so the $A$-action is

$$
a *\left(x_{1}, x_{2}, \cdots, x_{m}\right)^{T}=\phi(a) \cdot\left(x_{1}, x_{2}, \cdots, x_{m}\right)^{T}
$$

for $a \in A$ and $x_{1}, \cdots, x_{m} \in M$.

Definition 1.8. Let $A$ be a connected graded algebra. A graded algebra homomorphism $\sigma=\left(\sigma_{i j}\right): A \rightarrow$ $\mathbb{M}_{m}(A)$ is called invertible if there is a graded algebra homomorphism $\varphi=\left(\varphi_{i j}\right): A \rightarrow \mathbb{M}_{m}(A)$ satisfying the following conditions:

$$
\sum_{k=1}^{m} \varphi_{j k} \circ \sigma_{i k}=\left\{\begin{array}{ll}
\operatorname{id}_{A}, & \text { if } i=j, \\
0, & \text { if } i \neq j,
\end{array} \text { and } \sum_{k=1}^{m} \sigma_{k j} \circ \varphi_{k i}= \begin{cases}\mathrm{id}_{A}, & \text { if } i=j \\
0, & \text { if } i \neq j\end{cases}\right.
$$

In this case, such $\varphi$ is unique, denoted by $\sigma^{-1}$.

Now assume $A$ is AS-regular of type $(h, l)$. Let $\sigma: A \rightarrow \mathbb{M}_{m}(A)$ be a graded invertible algebra homomorphism. Write $\varphi=\sigma^{-1}$.

Choose a minimal free resolution of trivial module ${ }_{A} k$, that is, $P \cdot \stackrel{\varepsilon_{A}}{\longrightarrow}{ }_{A} k$. Let graded vector space $U=k^{\oplus m}$. One obtains two quasi-isomorphisms

$$
\varepsilon_{A}^{\oplus m}: P^{\cdot} \otimes U \stackrel{\varepsilon_{A} \otimes U}{\longrightarrow}{ }_{A} k \otimes U \cong{ }_{A} k^{\oplus m}, \quad \text { and } \quad \varepsilon_{A}^{\oplus m}:{ }^{\varphi}(P \cdot \otimes U) \stackrel{\varepsilon_{A} \otimes U}{\longrightarrow}{ }_{A} k \otimes U \cong{ }_{A} k^{\oplus m},
$$

where $i$-th term of ${ }^{\varphi}(P \cdot \otimes U)$ is the $\varphi$-twisted graded $A$-module ${ }^{\varphi}\left(P^{-i} \otimes U\right)$, since ${ }^{\varphi}\left(k^{\oplus m}\right) \cong k^{\oplus m}$ as graded $A$-modules. 
It is not hard to check that each ${ }^{\varphi}\left(P^{-i} \otimes U\right)$ is still a free $A$-module for $0 \leq i \leq h_{A}$. Hence, $P \otimes U$ and $\varphi(P \cdot \otimes U)$ are both minimal free resolutions of ${ }_{A} k^{\oplus m}$, and the direct sum of $m$ copies of Ext-algebra of $A$

$$
E(A)^{\oplus m} \cong \underline{\operatorname{Hom}}_{A}(P \cdot \otimes U, k) \cong \underline{\operatorname{Hom}}_{A}\left({ }^{\varphi}\left(P^{\cdot} \otimes U\right), k\right) .
$$

By Comparison Theorem, there exists an isomorphism of cochain complexes $\theta:{ }^{\varphi}\left(P^{\cdot} \otimes U\right) \rightarrow P^{\cdot} \otimes U$ in $\mathrm{Ch}(\mathrm{Gr} A)$ such that $\varepsilon_{A}^{\oplus m} \circ \theta=\varepsilon_{A}^{\oplus m}$. Then we have a bigraded map

$$
\underline{\operatorname{Hom}}_{A}(\theta, k): E(A)^{\oplus m} \rightarrow E(A)^{\oplus m} .
$$

In particular, $E^{h}(A)$ is one dimensional because of AS-regularity. Let $\omega^{*}$ be a basis of $E^{h}(A)$, and write $\left\{\omega_{i}^{*}=\left(0, \cdots, \omega^{*}, \cdots, 0\right)\right\}_{i=1}^{m}$ to be the canonical basis set of $\left(E^{h}(A)\right)^{\oplus m}$. When restricted on $\left(E^{h}(A)\right)^{\oplus m}$, $\underline{\operatorname{Hom}}_{A}(\theta, k)$ provides a matrix $H \in \mathbb{M}_{m}(k)$ satisfying

$$
\underline{\operatorname{Hom}}_{A}(\theta, k)\left(\omega_{1}^{*}, \cdots, \omega_{m}^{*}\right)^{T}=H\left(\omega_{1}^{*}, \cdots, \omega_{m}^{*}\right)^{T} .
$$

We say the matrix $H$ is the homological determinant of $\sigma$, denoted by hdet $\sigma$.

Remark 1.9. If $m=1$, the definition of homological determinant here is coincident with the one given in [6]. If $m=2$ and $A$ is Koszul algebra, it is also the same to the one in [21].

Note that $\theta$ is an isomorphism of cochain complexes, we have an immediate result.

Proposition 1.10. The homological determinant hdet $\sigma$ is an invertible matrix.

\section{Twisted TENSOR PRODUCTS}

In this section, we review definitions and some basic facts mainly from [3, 4, 18], and discuss some homological properties of twisted tensor products. We always restrict ourselves on (bi-)graded cases.

2.1. Basic facts. Let $A$ and $B$ be two connected graded algebras, and $\tau: B \otimes A \rightarrow A \otimes B$ be a graded linear map. Define $A \otimes^{\tau} B$ equals $A \otimes B$ as graded vector spaces with the multiplication given by

$$
m_{A \otimes^{\tau} B}=\left(m_{A} \otimes m_{B}\right)\left(\mathrm{id}_{A} \otimes \tau \otimes \mathrm{id}_{B}\right),
$$

where $m_{A}, m_{B}$ are products of $A, B$ respectively. The elements can be presented by $a \otimes^{\tau} b$ in $A \otimes^{\tau} B$ for any $a \in A, b \in B$. In general, $A \otimes^{\tau} B$ is not an associative graded algebra.

Definition 2.1. We say $A \otimes^{\tau} B$ is a twisted tensor product, if it is associative and $1_{A} \otimes^{\tau} 1_{B}$ is the unit. In this case, $\tau$ is called a graded twisting map.

We adopt the same notation for twisted tensor products to the one used in [17], by writing twisting maps as superscripts to avoid some confusion with the common tensor products over general rings. The usual tensor product is a special kind of twisted tensor products when the graded twisting map $\tau$ is chosen to be the filliping map, and we still use the notation $\otimes$ without superscript for it. The name " $R$-smash product" is used in [3, 12, 18], which is the same to twisted tensor product, we prefer the later one here.

To guarantee the unit condition, we need a normal assumption on $\tau$ : for all $a \in A, b \in B$,

$$
\tau\left(b \otimes 1_{A}\right)=1_{A} \otimes b \quad \text { and } \quad \tau\left(1_{B} \otimes a\right)=a \otimes 1_{B} .
$$


There is a necessary and sufficient condition for twisted tensor products ([3, Theorem 2.5], [4, Remark 2.4]): $\tau$ is a graded twisting map if and only if $\tau$ is normal and quasitriangular, that is,

$$
\begin{aligned}
& \left(\mathrm{id}_{A} \otimes m_{B}\right)\left(\tau \otimes \mathrm{id}_{B}\right)\left(\mathrm{id}_{B} \otimes \tau\right)=\tau\left(m_{B} \otimes \mathrm{id}_{A}\right), \\
& \left(m_{A} \otimes \mathrm{id}_{B}\right)\left(\mathrm{id}_{A} \otimes \tau\right)\left(\tau \otimes \mathrm{id}_{A}\right)=\tau\left(\mathrm{id}_{B} \otimes m_{A}\right) .
\end{aligned}
$$

In fact, there is a functor $A \otimes^{\tau}-: \operatorname{Gr} B \rightarrow \operatorname{Gr}\left(A \otimes^{\tau} B\right)$ defined as follows. For any graded module $M \in \mathrm{Gr} B, A \otimes^{\tau} M$ is the graded vector space $A \otimes M$ equipped with the scalar multiplication given by

$$
\left(m_{A} \otimes \lambda_{M}\right) \circ\left(\mathrm{id}_{A} \otimes \tau \otimes \mathrm{id}_{M}\right),
$$

where $m_{A}$ is the multiplication of $A$ and $\lambda_{M}$ is the scalar multiplication of $M$ as a graded $B$-module. The action of $A \otimes^{\tau}-$ on morphisms is the same to the map $A \otimes-$ on morphisms.

Similarly, we can define a functor $-\otimes^{\tau} B: \operatorname{Gr} A^{o} \rightarrow \operatorname{Gr}\left(A \otimes^{\tau} B\right)^{o}$.

Lemma 2.2. There are two pairs of natural isomorphic functors

$$
\begin{array}{llll}
A \otimes^{\tau}-\cong\left(A \otimes^{\tau} B\right) \otimes_{B}-: & \operatorname{Gr} B & \rightarrow & \operatorname{Gr}\left(A \otimes^{\tau} B\right), \\
-\otimes^{\tau} B \cong-\otimes_{A}\left(A \otimes^{\tau} B\right): & \operatorname{Gr} A^{o} & \rightarrow & \operatorname{Gr}\left(A \otimes^{\tau} B\right)^{o} .
\end{array}
$$

In order to obtain some nice combinatorial and homological properties for the twisted tensor product $A \otimes^{\tau} B$, we always restrict ourselves to the following situation.

Let $A=k\left\langle x_{1}, \cdots, x_{n}\right\rangle /\left(G_{A}\right)$ and $B=k\left\langle y_{1}, \cdots, y_{m}\right\rangle /\left(G_{B}\right)$ be two connected graded algebras. Since $\tau$ is just a graded linear map, we require $\tau$ being determined by its action on the generating set of $B \otimes A$ as in [18]. That is, we ask the linear map $\tau$ has form of

$$
\tau\left(y_{j} \otimes x_{i}\right)=\sum_{t=1}^{m} a_{i j t} \otimes y_{t}+a_{i j} \otimes 1,
$$

where $a_{i j t}, a_{i j} \in A$ and $i=1, \cdots, n, j=1, \cdots, m$. Following the quasitriangular condition for graded linear map $\tau$, we obtain an algebra homomorphism $\sigma=\left(\sigma_{i j}\right): A \rightarrow \mathbb{M}_{m}(A)$ and a $\sigma$-derivation $\delta=\left(\delta_{i}\right): A \rightarrow A^{\oplus m}$ such that

$$
\tau\left(y_{i} \otimes a\right)=\sum_{t=1}^{m} \sigma_{i t}(a) \otimes y_{s}+\delta_{i}(a) \otimes 1, \quad \forall a \in A, i=1, \cdots, m .
$$

In the sequel, we write such form $\tau=(\sigma, \delta)$.

Example 2.3. For Ore extensions $A[z ; \sigma, \delta]$ or double Ore extensions $A_{P}\left[y_{1}, y_{2} ; \sigma, \delta\right]$, they are all special cases of twisted tensor products if we define $\tau=(\sigma, \delta)$ (see Example 5.1 and Example 5.2 for detail).

Moreover, if we assume the graded algebra homomorphism $\sigma: A \rightarrow \mathbb{M}_{m}(A)$ is invertible in the sense of Definition 1.8, there exists a graded linear map $\tau^{-1}: A \otimes B \rightarrow B \otimes A$, which is the inverse of $\tau$, satisfying

$$
\tau^{-1}\left(a \otimes y_{i}\right)=\sum_{s=1}^{m} y_{s} \otimes \varphi_{s i}(a)+1 \otimes \delta_{i}^{\prime}(a),
$$

where $a \in A, \delta_{i}^{\prime}=-\sum_{j=1}^{m} \delta_{j} \circ \varphi_{j i}$ and $\varphi=\sigma^{-1}$ for $i=1, \cdots, m$. Following the quasitriangular condition, one obtains $\tau^{-1}$ is also a graded twisting map, equivalently, $B \otimes^{\tau^{-1}} A$ is a twisted tensor product.

Lemma 2.4. [18, Proposition 2.12] As graded algebras, $A \otimes^{\tau} B \cong B \otimes^{\tau^{-1}} A$.

Note that the definition of twisted tensor products can be given for bigraded algebras similarly. We close this subsection by exhibit the factorization theorem for twisted tensor products of bigraded algebras. 
Theorem 2.5. [3] Theorem 2.10] Let A, B and C be bigraded algebras. Then the following two conditions are equivalent

(a) As bigraded algebras, $C \cong A \otimes^{\tau} B$ for some bigraded twisting map $\tau: B \otimes A \rightarrow A \otimes B$;

(b) There exist bigraded algebra homomorphisms $f_{A}: A \rightarrow C$ and $f_{B}: B \rightarrow C$ such that

$$
m_{C} \circ\left(f_{A} \otimes f_{B}\right): A \otimes B \rightarrow C
$$

is an isomorphism of bigraded vector spaces, where $m_{C}$ is the multiplication of $C$.

In this case, the bigraded twisting map $\tau=\left(m_{C} \circ\left(f_{A} \otimes f_{B}\right)\right)^{-1} \circ m_{C} \circ\left(f_{B} \otimes f_{A}\right)$.

2.2. Minimal free resolutions. The main goal of this subsection is to construct a minimal free resolution for the trivial module of a twisted tensor product of two connected graded algebras. In order to obtain some interesting homological relations between $A, B$ and the twisted tensor product $A \otimes^{\tau} B$, we always make the discussion with the following hypotheses throughout this paper, unless otherwise stated.

\section{Hypothesis 2.6.}

(a) Let $A$ be a connected Ext-finite graded algebra, and $\left\{x_{1}, \cdots, x_{n}\right\}$ be a minimal generating set of A;

(b) Let $B$ be a connected Ext-finite graded algebra admitting a pure resolution, and $\left\{y_{1}, \cdots, y_{m}\right\}$ be a minimal generating set of $B$;

(c) Let $\tau=(\sigma, 0): B \otimes A \rightarrow A \otimes B$ be a graded twisting map, where $\sigma: A \rightarrow \mathbb{M}_{m}(A)$ is a graded algebra morphism.

Under the hypotheses above, there are two graded algebra injections

$$
\begin{aligned}
\iota_{A}: A & \rightarrow A \otimes^{\tau} B & \iota_{B}: B & \rightarrow A \otimes^{\tau} B \\
a & \mapsto a \otimes^{\tau} 1_{B}, & b & \mapsto 1_{A} \otimes^{\tau} b,
\end{aligned}
$$

and two graded algebra surjections

$$
\begin{aligned}
& \pi_{A}: A \otimes^{\tau} B \quad \rightarrow \quad A \\
& a \otimes^{\tau} b \quad \mapsto a \cdot \varepsilon_{B}(b), \\
& \pi_{B}: A \otimes^{\tau} B \quad \rightarrow \quad B \\
& a \otimes^{\tau} b \quad \mapsto \varepsilon_{A}(a) \cdot b,
\end{aligned}
$$

where $\varepsilon_{A}: A \rightarrow k$ and $\varepsilon_{B}: B \rightarrow k$ are the canonical augmentations. Clearly,

$$
\pi_{A} \circ \iota_{A}=\mathrm{id}_{A} \quad \text { and } \quad \pi_{B} \circ \iota_{B}=\mathrm{id}_{B} .
$$

By Lemma 1.2, we have an immediate result.

Corollary 2.7. The bigraded algebra homomorphisms $E\left(\pi_{A}\right): E(A) \rightarrow E\left(A \otimes^{\tau} B\right)$ and $E\left(\pi_{B}\right): E(B) \rightarrow$ $E\left(A \otimes^{\tau} B\right)$ are both injective.

To show the bigraded algebra homomorphisms $E\left(\pi_{A}\right), E\left(\pi_{B}\right)$ and the Yoneda product of their images, we begin with minimal free resolutions of trivial modules. For convenience, write $C:=A \otimes^{\tau} B$.

Remark 2.8. The graded algebra homomorphisms $\pi_{A}$ and $\pi_{B}$ make all graded $A$-modules (resp. right $A$ modules) and graded $B$-modules (resp. right $B$-modules) be graded $C$-modules (resp. right $C$-modules). In most discussion of this paper, we will transfer objects and morphisms in $\operatorname{Gr} A$ and $\mathrm{Gr} B$ ( $\operatorname{resp}$. $\mathrm{Ch}(\mathrm{Gr} A)$ and $\mathrm{Ch}(\mathrm{Gr} B))$ to ones in $\mathrm{Gr} C$ (resp. $\mathrm{Ch}(\mathrm{Gr} C))$ automatically. 
In the sequel, we fix a minimal free resolution of ${ }_{A} k$ of the following form:

$$
P^{*}=\left(\cdots \stackrel{d_{P}^{-4}}{\longrightarrow} A \otimes V_{3} \stackrel{d_{P}^{-3}}{\longrightarrow} A \otimes V_{2} \stackrel{d_{P}^{-2}}{\longrightarrow} A \otimes V_{1} \stackrel{d_{P}^{-1}}{\longrightarrow} A \rightarrow 0 \rightarrow \cdots\right) \quad \stackrel{\varepsilon_{A}}{\longrightarrow} \quad{ }_{A} k,
$$

where $V_{i}$ is a graded vector space for $i \geq 1$. Let $V_{0}=k$ and $V_{i}=0$ for $i<0$.

Since $B$ has a pure resolution, we may fix a minimal free resolution of ${ }_{B} k$ of the form:

$$
Q=\left(\cdots \stackrel{d_{Q}^{-4}}{\longrightarrow} B \otimes W_{3} \stackrel{d_{Q}^{-3}}{\longrightarrow} B \otimes W_{2} \stackrel{d_{Q}^{-2}}{\longrightarrow} B \otimes W_{1} \stackrel{d_{Q}^{-1}}{\longrightarrow} B \rightarrow 0 \rightarrow \cdots\right) \quad \stackrel{\varepsilon_{B}}{\longrightarrow} \quad{ }_{B} k,
$$

satisfying that each $W_{i}=k\left(-l_{i}\right)^{\oplus s_{i}}$ with $l_{i}, s_{i} \geq 1$ and $s_{1}=m$, and $d_{Q}^{-1}$ assigns $1 \otimes e_{j} \in B \otimes W_{1}$ to the coset of $y_{j}$ in $B$ for $j=1, \cdots, m$, where $\left\{e_{j}\right\}_{j=1}^{m}$ is the canonical coordinate basis of $W_{1}$. Write $W_{0}=k$ and $W_{i}=0$ for $i<0$.

Since the functor $A \otimes^{\tau}-$ is exact and preserves projective graded modules by Lemma 2.2, one obtains a minimal free resolution $F^{*}=A \otimes^{\tau} Q^{\cdot}$ of $A$ in $\operatorname{Gr} C$ of the form:

$$
F^{\cdot}=\left(\cdots \stackrel{d_{F}^{-4}}{\longrightarrow} C \otimes W_{3} \stackrel{d_{F}^{-3}}{\longrightarrow} C \otimes W_{2} \stackrel{d_{F}^{-2}}{\longrightarrow} C \otimes W_{1} \stackrel{d_{F}^{-1}}{\longrightarrow} C \rightarrow 0 \rightarrow \cdots\right) \quad \stackrel{\pi_{A}}{\longrightarrow} \quad C_{C} A .
$$

It is worthwhile to notice that the differential $d_{F}^{-i}=A \otimes^{\tau} d_{Q}^{-i}$ for each $i \geq 0$.

The next key step is to make the minimal free resolution $F$ of ${ }_{C} A$ be quasi-isomorphic to ${ }_{C} A_{A}$ in $\mathrm{Ch}\left(\mathrm{Gr}\left(C \otimes A^{o}\right)\right)$. To this end, we use the assumption on $B$, that it has a pure resolution, to endow each term of resolution $F^{*}$ with a graded $\phi$-twisted right $A$-module structure for some graded algebra homomorphism $\phi$ from $A$ to a matrix algebra over $A$.

Theorem 2.9. [18, Theorem 3.7] There exists a sequence of graded algebra homomorphisms $\phi_{n}: A \rightarrow$ $\mathbb{M}_{S_{n}}(A)$ for $n \geq 2$, such that $\pi_{A}: F_{\dot{\phi}} \rightarrow A$ is a quasi-isomorphism in $\operatorname{Ch}\left(\mathrm{Gr}\left(C \otimes A^{o}\right)\right)$, where

$$
F_{\dot{\phi}}^{*}=\cdots \stackrel{d_{F}^{-4}}{\longrightarrow}\left(C \otimes W_{3}\right)^{\phi_{3}} \stackrel{d_{F}^{-3}}{\longrightarrow}\left(C \otimes W_{2}\right)^{\phi_{2}} \stackrel{d_{F}^{-2}}{\longrightarrow}\left(C \otimes W_{1}\right)^{\sigma} \stackrel{d_{F}^{-1}}{\longrightarrow} C \rightarrow 0 \rightarrow \cdots .
$$

Moreover, if $B$ is AS-regular algebra of type $\left(h_{B}, l_{B}\right)$ and $\sigma$ is invertible, then $F_{\phi}$ terminates at $\left(-h_{B}\right)$-th term, and $\phi_{h_{B}}$ is a graded automorphism of $A$.

Sketch of proof. We describe the construction of complex $F_{\phi}$ briefly, which may be useful in Example 2.15 and Example 5.3, and see [18] for the completed proof in a more general situation.

Firstly, it is obvious that the differential $d_{F}^{-1}$ becomes a graded right $A$-module homomorphism when the (-1)-th term of $F^{*}$ is endowed with a $\sigma$-twisted graded right $A$-module structure, because of the choice of $d_{F}^{-1}$. Then we obtain the sequence of graded algebra homomorphisms $\phi_{n}$ for $n \geq 2$ inductively. Write $\phi_{1}=\sigma$ and $\phi_{0}=\mathrm{id}_{A}$.

Suppose we have got the desired graded algebra homomorphisms $\left\{\phi_{j}\right\}_{j=1}^{i-1}$ where $i \geq 2$. For $\phi_{i}: A \rightarrow$ $\mathbb{M}_{s_{i}}(A)$, we consider the following exact complex

$$
C \otimes W_{i} \stackrel{d_{F}^{-i}}{\longrightarrow}\left(C \otimes W_{i-1}\right)^{\phi_{i-1}} \stackrel{d_{F}^{-i+1}}{\longrightarrow}\left(C \otimes W_{i-2}\right)^{\phi_{i-2}} .
$$

Write the canonical coordinate basis of $W_{i}=k\left(-l_{i}\right)^{\oplus s_{i}}$ to be $\left\{e_{j}^{i}\right\}_{j=1}^{s_{i}}$. For any $a \in A$ and $j=1, \cdots, s_{i}$, the element $\left(d_{F}^{-i}\left(1 \otimes e_{j}^{i}\right)\right) * a$ lies in the image of $d_{F}^{-i}$, since $d_{F}^{-i+1}$ is a graded right $A$-module homomorphism, say

$$
d_{F}^{-i}\left(\sum_{p=1}^{s_{i}}\left(c_{j p} \otimes e_{p}^{i}\right)\right)=\left(d_{F}^{-i}\left(1 \otimes e_{j}^{i}\right)\right) * a,
$$


for some $c_{j p} \in C$ and $p=1, \cdots, s_{i}$. Note that $d_{F}^{-i}=A \otimes^{\tau} d_{Q}^{-i}$, and $W_{i}$ and $W_{i-1}$ are both concentrated in one degree. By an argument about degrees of elements in both sides of the equation above, we have that each $c_{j p}$ has form $a_{j p} \otimes^{\tau} 1_{B}$, where $a_{j p} \in A$ for $p=1, \cdots, s_{i}$. Define $\phi_{i}=\left(\left(\phi_{i}\right)_{j p}\right): A \rightarrow \mathbb{M}_{s_{i}}(A)$ such that

$$
\left(\phi_{i}\right)_{j p}(a)=a_{j p},
$$

for $j, p=1, \cdots, s_{i}$. It can be checked $\phi_{i}$ is a well-defined graded algebra homomorphism, and make the differential $d_{F}^{-i}:\left(C \otimes W_{i}\right)^{\phi_{i}} \rightarrow\left(C \otimes W_{i-1}\right)^{\phi_{i-1}}$ be a morphism in $\operatorname{Gr}\left(C \otimes A^{o}\right)$.

Proposition 2.10. The morphism of cochain complexes $\varepsilon_{C}: F_{\dot{\phi}} \otimes_{A} P \cdot \rightarrow{ }_{C} k$ is a quasi-isomorphism. As a consequence, $F_{\dot{\phi}} \otimes_{A} P$. is a minimal free resolution of ${ }_{C} k$.

Proof. The augmentation $\varepsilon_{C}$ of $C$ equals the composition of complexes morphisms

$$
F_{\phi}^{\cdot} \otimes_{A} P \cdot \stackrel{\pi_{A} \otimes_{A} P}{\simeq}\left({ }_{C} A_{A}\right) \otimes_{A} P \cdot \stackrel{A \otimes_{A} \varepsilon_{A}}{\simeq}\left({ }_{C} A_{A}\right) \otimes_{A}\left({ }_{A} k\right) \cong{ }_{C} k,
$$

and it is a quasi-isomorphism. The minimality is clear.

The complex $F_{\dot{\phi}}$ also helps us acquire the following result about AS-regularity of twisted tensor products.

Theorem 2.11. [18, Theorem 0.3] Let A be AS-regular of type $\left(h_{A}, l_{A}\right)$ and $B$ be AS-regular of type $\left(h_{B}, l_{B}\right)$. Let $\tau=(\sigma, \delta): B \otimes A \rightarrow A \otimes B$ be a graded twisting map. If $B$ has a pure resolution and $\sigma$ is invertible, then $A \otimes^{\tau} B$ is $A S$-regular of type $\left(h_{A}+h_{B}, l_{A}+l_{B}\right)$.

2.3. Determinant. For a graded algebra homomorphism $\sigma=\left(\sigma_{i j}\right): A \rightarrow \mathbb{M}_{m}(A), \sigma$ is an $m \times m$ matrix consisting of graded linear maps $\left\{\sigma_{i j}: A \rightarrow A\right\}_{i, j=1}^{m}$. Classical determinant is an important invariant for a matrix, and we will give a generalized definition of determinant for such graded invertible algebra homomorphisms in the sense of Definition 1.8

Definition 2.12. Let $A, B$ be two connected graded algebras, and $\sigma: A \rightarrow \mathbb{M}_{m}(A)$ be a graded invertible algebra homomorphism where $m$ is the number of elements in the minimal generating set of $B$. If graded linear map $\tau=(\sigma, 0): B \otimes A \rightarrow A \otimes B$ is a graded twisting map and there exists a graded algebra automorphism $v$ of $A$ satisfying

$$
\underline{\operatorname{Ext}}_{A \otimes^{\tau} B}^{i}\left(A, A \otimes^{\tau} B\right) \cong \begin{cases}0 & i \neq d, \\ { }^{v} A(l) & i=d,\end{cases}
$$

as graded $A^{e}$-modules for some integers $d$ and $l$, we say $v$ is the determinant of $\sigma$, denoted by $\operatorname{det} \sigma$.

Since $A$ is connected, the determinant $\operatorname{det} \sigma$ is unique whenever it exists. The definition of determinant above is a generalization of the one given for the case of double Ore extensions by [20, Theorem 0.4]. However, the determinant does not exist in general. In the following, we focus on the interested case in which the determinant always exists.

We assume connected graded algebras $A, B$ and the graded linear map $\tau=(\sigma, 0): B \otimes A \rightarrow A \otimes B$ satisfy Hypothesis 2.6 So $\sigma$ is a graded algebra homomorphism from $A$ to $\mathbb{M}_{m}(A)$. In addition, assume $B$ is AS-regular of type $\left(h_{B}, l_{B}\right)$ and $\sigma$ is invertible. Now the complex (RC2) in Theorem 2.9 becomes

(RC3) $F_{\dot{\phi}}^{\cdot}=\cdots \rightarrow 0 \rightarrow\left(C \otimes W_{h_{B}}\right)^{\phi_{h_{B}}} \stackrel{d_{F}^{-h_{B}}}{\longrightarrow}\left(C \otimes W_{h_{B}-1}\right)^{\phi_{h_{B}-1}} \stackrel{d_{F}^{-h_{B}+1}}{\longrightarrow} \cdots \stackrel{d_{F}^{-2}}{\longrightarrow}\left(C \otimes W_{1}\right)^{\sigma} \stackrel{d_{F}^{-1}}{\longrightarrow} C \rightarrow 0 \rightarrow \cdots$, where $W_{h_{B}}=k\left(-l_{B}\right)$ and $\phi_{h_{B}}$ is a graded automorphism of $A$. 
The determinant of $\sigma$ is related to the cohomology of cochain complex $\underline{\operatorname{Hom}}_{C}\left(F_{\phi}^{\cdot}, C\right)$. Before computing the cohomologies, we transfer the Hom-set to the symmetrical version $B \otimes^{\tau^{-1}} A$.

Let $\psi: A \rightarrow \mathbb{M}_{p}(A)$ be a graded algebra homomorphism and $W=k(t)^{\oplus p}$ be a graded vector space for some integers $t$ and $p$. It is obvious, $\underline{\operatorname{Hom}}_{A \otimes^{\tau} B}\left(\left(A \otimes^{\tau} B\right) \otimes W, A \otimes^{\tau} B\right) \cong W^{*} \otimes\left(A \otimes^{\tau} B\right)$ as graded $A^{e}$-modules. With additional $\psi$-twisted graded $A$-module structures, we have following isomorphisms in $\operatorname{Gr} A^{e}$,

$$
\begin{aligned}
\underline{\operatorname{Hom}}_{A \otimes^{\tau} B}\left(\left(\left(A \otimes^{\tau} B\right) \otimes W\right)^{\psi}, A \otimes^{\tau} B\right) & \cong \psi\left(W^{*} \otimes\left(A \otimes^{\tau} B\right)\right) \\
& \cong \psi\left(W^{*} \otimes\left(B \otimes^{\tau^{-1}} A\right)\right) \\
& \cong \psi\left(\left(W^{*} \otimes B\right) \otimes^{\tau^{-1}} A\right) \\
& \cong \psi\left(\underline{\operatorname{Hom}}_{B}(B \otimes W, B) \otimes^{\tau^{-1}} A\right)
\end{aligned}
$$

where the second isomorphism comes from Lemma 2.4 the third isomorphism follows from Lemma 2.2 and the $\psi$-twisted graded $A$-module structure of $\psi\left(\underline{\operatorname{Hom}}_{B}(B \otimes W, B) \otimes \tau^{\tau^{-1}} A\right)$ is induced by the one of $\psi\left(\left(B \otimes^{\tau^{-1}} A\right) \otimes W^{*}\right)$. Now we have an isomorphism as below.

Lemma 2.13. As graded $A^{e}$-modules, $\underline{\operatorname{Hom}}_{A \otimes^{\tau} B}\left(\left(\left(A \otimes^{\tau} B\right) \otimes W\right)^{\psi}, A \otimes^{\tau} B\right) \cong \psi\left(\underline{\operatorname{Hom}}_{B}(B \otimes W, B) \otimes^{\tau^{-1}} A\right)$.

Then we can prove the main result of this subsection.

Lemma 2.14. The determinant $\operatorname{det} \sigma=\phi_{h_{B}}$.

Proof. Applying the functor $\underline{\operatorname{Hom}}_{C}(-, C)$ to the complex $F_{\dot{\phi}} \sqrt{\mathrm{RC} 3}$, we have a commutative diagram by Lemma 2.13

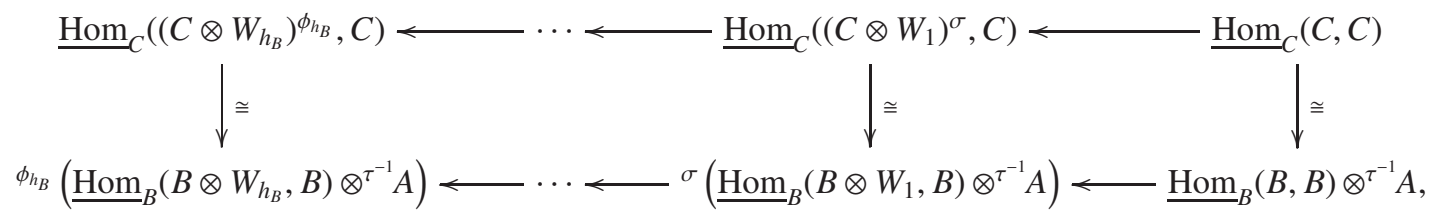

and the bottom cochain complex is just $\underline{\operatorname{Hom}}_{B}(Q, B) \otimes{ }^{\tau^{-1}} A$, where $Q \cdot$ is the minimal resolution of ${ }_{B} k$, when forgetting the graded left $A$-module structures. Since the functor $-\otimes^{\tau^{-1}} A$ is exact and $B$ is AS-regular, the result follows.

The following is an easy example to explain why we use the word "determinant".

Example 2.15. Let $A$ be a connected graded algebra and $B=k\left[y_{1}, \cdots, y_{m}\right]$ be a polynomial algebra with $\operatorname{deg} y_{i}=l$ where $l \geq 1$. Let $\sigma: A \rightarrow \mathbb{M}_{m}(A)$ be a graded invertible algebra homomorphism such that $A \otimes^{\tau} B$ is a twisted tensor product where $\tau=(\sigma, 0)$. Polynomial algebra $B$ is Koszul AS-regular of type $(m, m l)$, so the determinant of $\sigma$ exists and needs to construct the complex $F_{\phi}^{*}$ by Lemma 2.14 Firstly, one obtains a minimal free resolution of ${ }_{B} k$

$$
\left(\cdots \rightarrow 0 \rightarrow B \otimes W_{m} \stackrel{d^{-m}}{\longrightarrow} B \otimes W_{m-1} \stackrel{d^{-m+1}}{\longrightarrow} \cdots \stackrel{d^{-2}}{\longrightarrow} B \otimes W_{1} \stackrel{d^{-1}}{\longrightarrow} B \rightarrow 0 \rightarrow \cdots\right) \stackrel{\varepsilon_{B}}{\longrightarrow} \quad{ }_{B} k,
$$

where each graded vector space $W_{i}=k^{\oplus t_{i}}(-i l)$ and $t_{i}=\left(\begin{array}{c}m \\ i\end{array}\right)$ with a canonical basis $\left\{e_{j_{1} j_{2} \cdots j_{i}} \mid\left(j_{1}, \cdots, j_{i}\right) \in J_{i}\right\}$, index set $J_{i}=\left\{\left(j_{1}, \cdots, j_{i}\right) \mid j_{1}, \cdots, j_{i}=1, \cdots, m\right.$ and $\left.j_{1}<j_{2}<\cdots<j_{i}\right\}$, and each differential $d^{-i}$ satisfies

$$
d^{-i}\left(1 \otimes e_{j_{1} j_{2} \cdots j_{i}}\right)=\sum_{u=1}^{i}(-1)^{(u-1)} y_{j_{u}} \otimes e_{j_{1} \cdots j_{u-1} j_{u+1} \cdots j_{i}} .
$$


To make the following steps clear, it is necessary to sort the elements in each index set $J_{i}$. For any $\left(j_{1}, \cdots, j_{i}\right)$ and $\left(j_{1}^{\prime}, \cdots, j_{i}^{\prime}\right)$ in $J_{i}$, we say $\left(j_{1}, \cdots, j_{i}\right)<\left(j_{1}^{\prime}, \cdots, j_{i}^{\prime}\right)$ if there exists a positive integer $t$ such that $j_{p}=j_{p}^{\prime}$ for $p<t$ and $j_{t}>j_{t}^{\prime}$. According to the ordering <, we define a sorting map $\chi_{i}: J_{i} \rightarrow\left\{1, \cdots,\left(\begin{array}{c}m \\ i\end{array}\right)\right\}$ by sending the largest element $(1,2, \cdots, i)$ to 1 , second largest element $(1, \cdots, i-1, i+1)$ to 2 , and so forth.

The required complex can be acquired by acting the functor $A \otimes^{\tau}-$ to the minimal resolution above $0 \rightarrow\left(\left(A \otimes^{\tau} B\right) \otimes W_{m}\right)^{\psi_{m}} \stackrel{A \otimes^{\tau} d^{-m}}{\longrightarrow}\left(\left(A \otimes^{\tau} B\right) \otimes W_{m-1}\right)^{\psi_{m-1}} \stackrel{A \otimes^{\tau} d^{-m+1}}{\longrightarrow} \cdots \stackrel{A \otimes^{\tau} d^{-2}}{\longrightarrow}\left(\left(A \otimes^{\tau} B\right) \otimes W_{1}\right)^{\sigma} \stackrel{A \otimes^{\tau} d^{-1}}{\longrightarrow} B \rightarrow 0$, where $\psi_{i}=\left(\left(\psi_{i}\right)_{p q}\right): A \rightarrow \mathbb{M}_{t_{i}}(A)$ is a graded algebra homomorphism with entry $\left(\psi_{i}\right)_{p q}$ being the classical determinant of the submatrix formed by the $\chi_{i}^{-1}(p)$-th rows and $\chi_{i}^{-1}(q)$-th columns of $\sigma=\left(\sigma_{i j}\right)$. Using the map $\chi_{i}$ to sort the basis $\left\{e_{j_{1} j_{2} \cdots j_{i}}\right\}_{J_{i}}$ of $W_{i}$, then the graded $\psi_{i}$-twisted right $A$-module structure is clear for $i=2, \cdots, m$.

In particular, $\psi_{m}$ is just the classical determinant of the matrix $\sigma=\left(\sigma_{i j}\right)$. In other side, $\psi_{m}=\operatorname{det} \sigma$ by Lemma 2.14 That is to say, the classical determinant of matrix coincides with the one defined above in this case. Thus the determinant given in Definition 2.12 is considered as a generalization of classical determinants for matrices.

\section{EXT-ALGEBRAS OF TWISTED TENSOR PRODUCTS}

In this section, we focus on the Yoneda products of Ext-algebras of twisted tensor products and prove the main result Theorem $\mathrm{A}$, which is a more general context of [12, Theorem 3.7]. Let $A, B$ be connected graded algebras and graded linear map $\tau=(\sigma, 0): B \otimes A \rightarrow A \otimes B$ satisfy Hypothesis 2.6 We adopt the notations used in Section 2. Write $C:=A \otimes^{\tau} B$.

The minimal resolutions of ${ }_{A} k,{ }_{B} k$ and ${ }_{C} A_{A}$ are still denoted by $P^{*}, Q$ and $F_{\phi}^{*}$ respectively. By Proposition 2.10, $F_{\dot{\phi}} \otimes_{A} P \cdot$ is a minimal free resolution of ${ }_{C} k$. Starting from those minimal free resolutions, we have

$$
\begin{aligned}
& E(A)=\bigoplus_{i \geq 0} \underline{\operatorname{Hom}}_{A}\left(P^{-i}, k\right) \cong \bigoplus_{i \geq 0} V_{i}^{*}, \\
& E(B)=\bigoplus_{i \geq 0} \underline{\operatorname{Hom}}_{A}\left(Q^{-i}, k\right) \cong \bigoplus_{i \geq 0} W_{i}^{*}, \\
& E(C)=\bigoplus_{i \geq 0} \underline{\operatorname{Hom}}_{A}\left(\left(F_{\phi}^{*} \otimes_{A} P^{-}\right)^{-i}, k\right) \cong \bigoplus_{i \geq 0} \bigoplus_{p+q=i} W_{p}^{*} \otimes V_{q}^{*} .
\end{aligned}
$$

In the sequel, we identify the Ext-algebras $E^{i}(A), E^{i}(B)$ and $E^{i}(C)$ with $V_{i}^{*}, W_{i}^{*}$ and $\bigoplus_{p+q=i} W_{p}^{*} \otimes V_{q}^{*}$ for each $i \geq 0$ respectively.

Remark 3.1. We treat Ext-algebras $E(A), E(B)$ and $E(C)$ as graded $k$-dual spaces of some graded vector spaces to make notations uniform. However, we need to keep in mind that each element in such identifications for Ext-algebras has a corresponding representation through minimal free resolutions as in [12, Remark 3.3].

For any homogeneous element $f \in\left(V_{i}^{*}\right)_{-t}=E^{i}(A)_{-t}$, there is a corresponding morphism $\alpha:=P^{\cdot} \rightarrow$ $k(-t)[i]$ in $\mathrm{Ch}(\mathrm{Gr} A)$ induced by $\varepsilon_{A} \otimes f: A \otimes V_{i} \rightarrow{ }_{A} k(-t)$. Similarly, to the homogeneous elements in $E^{i}(B)$.

In other side, for any $g \otimes f \in\left(W_{j}^{*}\right)_{-s} \otimes\left(V_{i}^{*}\right)_{-t} \subseteq E^{i+j}(C)_{-s-t}$, there is a corresponding morphism $\gamma:=$ $F_{\dot{\phi}} \otimes_{A} P \cdot \rightarrow{ }_{C} k(-s-t)[i+j]$ in $\mathrm{Ch}(\mathrm{Gr} C)$ induced by

$$
\left(C \otimes W_{j}\right)^{\phi_{j}} \otimes_{A}\left(A \otimes V_{i}\right) \stackrel{\left(C \otimes W_{j}\right)^{\phi_{j} \otimes(A \otimes f)}}{\longrightarrow}\left(C \otimes W_{j}\right)(-t) \stackrel{\left(\pi_{B} \otimes W_{j}\right)(-t)}{\longrightarrow}\left(B \otimes W_{j}\right)(-t) \stackrel{\left(\varepsilon_{B} \otimes g\right)(-t)}{\longrightarrow} C k(-s-t) .
$$

Such representations are useful to prove results below. 
We are aimed at describing the connections between $E(A), E(B)$ and $E(C)$. For this purpose, we follow the action on morphisms of the functor $E(-)$ to characterize injections $E\left(\pi_{A}\right)$ and $E\left(\pi_{B}\right)$ through the minimal free resolutions $P \cdot Q \cdot$ and $F_{\phi}^{*} \otimes_{A} P$.

Since the algebra homomorphism $\pi_{A}: C \rightarrow A$ makes all $A$-modules be $C$-modules, so the cochain complex $P^{*}$ is also an object of $\operatorname{Ch}(\operatorname{Gr} C)$. And $\pi_{A}: F_{\dot{\phi}} \rightarrow A$ is a morphism of cochain complexes in $\mathrm{Ch}\left(\mathrm{Gr}\left(C \otimes A^{o}\right)\right)$, so we have a morphism in $\mathrm{Ch}(\mathrm{Gr} C)$ :

$$
\tilde{\pi}_{A}: F_{\phi}^{\cdot} \otimes_{A} P^{\cdot} \stackrel{\pi_{A} \otimes_{A} P}{\longrightarrow} A \otimes_{A} P^{*} \cong P^{\cdot},
$$

lifting $\pi_{A}$ such that $\varepsilon_{A} \circ \widetilde{\pi}_{A}=\varepsilon_{C}$.

In other side, the composition $A \stackrel{\iota_{A}}{\longrightarrow} C \stackrel{\pi_{B}}{\longrightarrow} B$ of graded algebra homomorphisms endows each graded right $B$-module with a trivial graded right $A$-module structure. Then $\left(B \otimes W_{i}\right)^{\phi_{i}} \cong B \otimes W_{i}$ as graded $B \otimes A^{o}$ modules. One obtains a morphism of cochain complexes $\widetilde{\pi}_{B}^{\prime}: F_{\dot{\phi}} \rightarrow Q$ in $\operatorname{Ch}\left(\operatorname{Gr}\left(C \otimes A^{o}\right)\right)$, of which each $i$-th component is $\pi_{B} \otimes W_{i}$, satisfying $\varepsilon_{A} \circ \pi_{A}=\varepsilon_{B} \circ \widetilde{\pi}_{B}^{\prime}$. Now we have a composition of morphisms lifting $\pi_{B}$ in $\mathrm{Ch}(\mathrm{Gr} C)$ :

$$
\tilde{\pi}_{B}: F_{\dot{\phi}} \otimes_{A} P \cdot \stackrel{\widetilde{\pi}_{B}^{\prime} \otimes_{A} P \cdot}{\longrightarrow} Q \cdot \otimes_{A} P \cdot \stackrel{\mathrm{pr}}{\longrightarrow} Q \cdot \otimes_{A} A \cong Q,
$$

where pr: $Q \cdot \otimes_{A} P \cdot \rightarrow Q \cdot \otimes_{A} A$ is a projection of cochain complexes by the minimality of $P$. Clearly, $\varepsilon_{B} \circ \widetilde{\pi_{B}}=\varepsilon_{C}$.

Lemma 3.2. For each $i \geq 0$, let $f \in V_{i}^{*}$ and $g \in W_{i}^{*}$, then

$$
E\left(\pi_{A}\right)(f)=1 \otimes f \in W_{0}^{*} \otimes V_{i}^{*} \subseteq E^{i}(C) \quad \text { and } \quad E\left(\pi_{B}\right)(g)=g \otimes 1 \in W_{i}^{*} \otimes V_{0}^{*} \subseteq E^{i}(C) .
$$

Proof. Without loss of generality, we assume $f \in\left(V_{i}^{*}\right)_{-t}$ is a homogeneous element. Let $\alpha: P \cdot \rightarrow{ }_{A} k(-t)[i]$ be the corresponding morphism of cochain complexes to $f$ by Remark 3.1 whose $(-i)$-th component is $\varepsilon_{A} \otimes f: A \otimes V_{i} \rightarrow{ }_{A} k(-t)$.

Following the definition of $E\left(\pi_{A}\right)$ above the Lemma 1.2, we have $E\left(\pi_{A}\right)(\alpha)=\alpha \circ \widetilde{\pi}_{A}$. However, the nonzero values of $(-i)$-th component of $\widetilde{\pi}_{A}$ only take from the direct summand $C \otimes_{A}\left(A \otimes V_{i}\right)$ of the $(-i)$-th term of $F_{\dot{\phi}} \otimes_{A} P$. Hence, the only nonzero part of $E\left(\pi_{A}\right)(\alpha)$ equals the composition of following morphisms

$$
C \otimes_{A}\left(A \otimes V_{i}\right) \stackrel{\pi_{A} \otimes_{A}\left(A \otimes V_{i}\right)}{\longrightarrow} A \otimes_{A}\left(A \otimes V_{i}\right) \cong A \otimes V_{i} \stackrel{\varepsilon_{A} \otimes f}{\longrightarrow} C k .
$$

Then $E\left(\pi_{A}\right)(f)=1 \otimes f$ corresponds to $E\left(\pi_{A}\right)(\alpha)$ when identifying $E^{i}(C)=\bigoplus_{j=0}^{i} W_{j}^{*} \otimes V_{i-j}^{*}$ by Remark 3.1 ,

Similarly, we have $E\left(\pi_{B}\right)(g)=g \otimes 1 \in W_{i}^{*} \otimes V_{0}^{*}$ by the morphism $\widetilde{\pi}_{B}$ in $\operatorname{Ch}(\operatorname{Gr} C)$.

Next step is to compute the Yoneda products of images of $E\left(\pi_{A}\right)$ and ones of $E\left(\pi_{B}\right)$ in $E(C)$. We divide it into three cases.

3.1. General case. We prove the first main result of this paper here. Before that, we describe one case of Yoneda products in $E(C)$ under Hypothesis 2.6 without additional conditions.

Lemma 3.3. Let $f \in V_{i}^{*}, g \in W_{j}^{*}$, then

$$
(g \otimes 1) \cdot(1 \otimes f)=(-1)^{i j} g \otimes f \in W_{j}^{*} \otimes V_{i}^{*} \subseteq E^{i+j}(C) .
$$


Proof. Without loss of generality, we assume $f \in\left(V_{i}^{*}\right)_{-t}$ and $g \in\left(W_{j}^{*}\right)_{-l_{j}}$ are both homogeneous elements. Write $\alpha: P \cdot \rightarrow{ }_{A} k(-t)[i]$ and $\beta: Q \cdot{ }_{B} k\left(-l_{j}\right)[j]$ to be the corresponding morphisms of $f$ and $g$ respectively. By the proof of Lemma 3.2 and Remark 3.1 $E\left(\pi_{A}\right)(\alpha): F_{\dot{\phi}} \otimes_{A} P \rightarrow{ }_{C} k(-t)[i]$ in $\mathrm{Ch}(\mathrm{Gr} C)$ corresponds to $1 \otimes f \in W_{0}^{*} \otimes V_{i}^{*}$ induced by

$$
\pi_{A} \otimes_{A}\left(\varepsilon_{A} \otimes f\right): C \otimes_{A}\left(A \otimes V_{i}\right) \rightarrow{ }_{C} k(-t),
$$

and $E\left(\pi_{B}\right)(\beta): F_{\dot{\phi}} \otimes_{A} P \cdot \rightarrow{ }_{C} k\left(-l_{j}\right)[j]$ in $\mathrm{Ch}(\mathrm{Gr} C)$ corresponds to $g \otimes 1 \in W_{j}^{*} \otimes V_{0}^{*}$ induced by

$$
\left(C \otimes W_{j}\right)^{\phi_{j}} \otimes_{A} A \cong C \otimes W_{j} \stackrel{\pi_{B} \otimes W_{j}}{\longrightarrow} B \otimes W_{j} \stackrel{\varepsilon_{B} \otimes g}{\longrightarrow} C k\left(-l_{j}\right) .
$$

By Comparison Theorem, choose a morphism $\widetilde{\alpha}: P^{*} \rightarrow P^{\cdot}(-t)[i]$ in $\mathrm{Ch}(\mathrm{Gr} A)$ such that $\varepsilon_{A}(-t)[i] \circ \widetilde{\alpha}=\alpha$. To be specific, the (-i)-th component of $\widetilde{\alpha}$ is $A \otimes f: A \otimes V_{i} \rightarrow A(-t)$.

Now consider the following commutative diagram in $\mathrm{Ch}(\mathrm{Gr} C)$ :

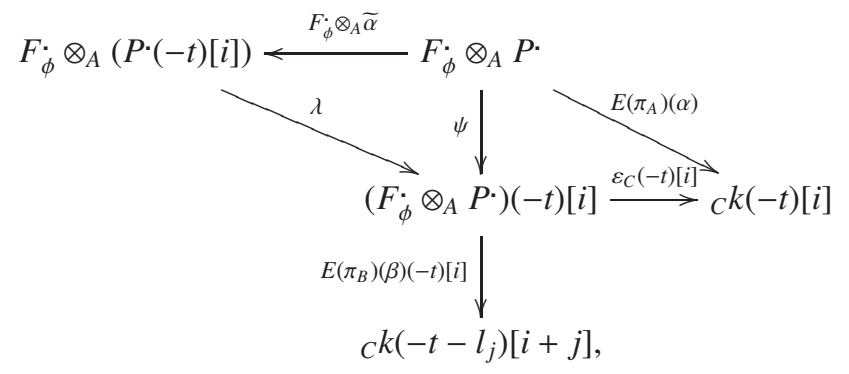

where $\lambda: F_{\phi}^{\cdot} \otimes_{A}\left(P^{\cdot}(-t)[i]\right) \rightarrow\left(F_{\phi}^{\cdot} \otimes_{A} P^{\cdot}\right)(-t)[i]$ acts on each summand $F_{\phi}^{-j} \otimes_{A} P^{-u}$ by multiplying $(-1)^{i j}$ for $j, u \geq 0$ and $\psi=\lambda \circ\left(F_{\phi}^{\cdot} \otimes_{A} \widetilde{\alpha}\right)$. It is easy to check $\varepsilon_{C}(-t)[i] \circ \psi=E\left(\pi_{A}\right)(\alpha)$.

Then $E\left(\pi_{B}\right)(\beta) \cdot E\left(\pi_{A}\right)(\alpha)=E\left(\pi_{B}\right)(\beta)(-t)[i] \circ \psi$, and the nonzero part equals composition of following morphisms

$\left(C \otimes W_{j}\right)^{\phi_{j}} \otimes_{A}\left(A \otimes V_{i}\right) \stackrel{(-1)^{i j}\left(C \otimes W_{j}\right)^{\phi_{j}} \otimes_{A}(A \otimes f)}{\longrightarrow}\left(C \otimes W_{j}\right)^{\phi_{j}}(-t) \stackrel{\left(\pi_{B} \otimes W_{j}\right)(-t)}{\longrightarrow}\left(B \otimes W_{j}\right)(-t) \stackrel{\left(\varepsilon_{B} \otimes g\right)(-t)}{\longrightarrow} C k\left(-t-l_{j}\right)$.

Hence, $(g \otimes 1)(1 \otimes f)=(-1)^{i j} g \otimes f$ by Remark 3.1

Theorem 3.4. There is a bigraded twisting map $\tau_{E}: E(A) \otimes E(B) \rightarrow E(B) \otimes E(A)$ satisfying

$$
m_{E(C)} \circ\left(E\left(\pi_{B}\right) \otimes E\left(\pi_{A}\right)\right) \circ \tau_{E}=m_{E(C)} \circ\left(E\left(\pi_{A}\right) \otimes E\left(\pi_{B}\right)\right),
$$

where $m_{E(C)}$ is the Yoneda product of $E(C)$, such that as bigraded algebras,

$$
E(C) \cong E(B) \otimes^{\tau_{E}} E(A) \text {. }
$$

Proof. By Corollary 2.7, we know that $E\left(\pi_{A}\right): E(A) \rightarrow E(C)$ and $E\left(\pi_{B}\right): E(B) \rightarrow E(C)$ are bigraded algebra homomorphisms. Write bigraded linear map

$$
\rho:=m_{E(C)} \circ\left(E\left(\pi_{B}\right) \otimes E\left(\pi_{A}\right)\right): E(B) \otimes E(A) \rightarrow E(C) .
$$

Following from Lemma 3.2 and Lemma 3.3, $\rho$ is an isomorphism of bigraded vector spaces. By Theorem 2.5 as bigraded algebras

$$
E(C) \cong E(B) \otimes^{\tau_{E}} E(A),
$$

where the bigraded twisting map $\tau_{E}=\rho^{-1} \circ m_{E(C)} \circ\left(E\left(\pi_{A}\right) \otimes E\left(\pi_{B}\right)\right): E(A) \otimes E(B) \rightarrow E(B) \otimes E(A)$. 
A good will is to give a specific formula for the bigraded twisting map $\tau_{E}$ in theorem above as the [12. Theorem 3.7]. Unfortunately, we cannot make our wish come true, since it is hard to know what $(1 \otimes f) \cdot(g \otimes 1)$ is where $f \in E(A)$ and $g \in E(B)$ in general. However, we may give a partially depict for such Yoneda products with AS-regularity in the following two subsections.

3.2. When $B$ is AS-regular. We assume $B$ is AS-regular of type $\left(h_{B}, l_{B}\right)$ and $A$ is generated in degree 1 in addition. In this case, let $A_{1}=V_{1}$ which is the graded vector space in minimal resolution (RA) of ${ }_{A} k$.

The minimal free resolution $\left(\mathrm{RB}\right.$ ) of trivial module ${ }_{B} k$ becomes

$$
Q:=\left(\cdots \rightarrow 0 \rightarrow B \otimes W_{h_{B}} \stackrel{d_{Q}^{-h_{B}}}{\longrightarrow} B \otimes W_{h_{B}-1} \stackrel{d_{Q}^{-h_{B}+1}}{\longrightarrow} \cdots \rightarrow B \otimes W_{1} \stackrel{d_{Q}^{-1}}{\longrightarrow} B \rightarrow 0 \rightarrow \cdots\right) \stackrel{\varepsilon_{B}}{\longrightarrow} \quad{ }_{B} k,
$$

where $W_{h_{B}}=k\left(-l_{B}\right)$. Recall the resolution (RC3) of ${ }_{C} A_{A}$ by Lemma 2.14,

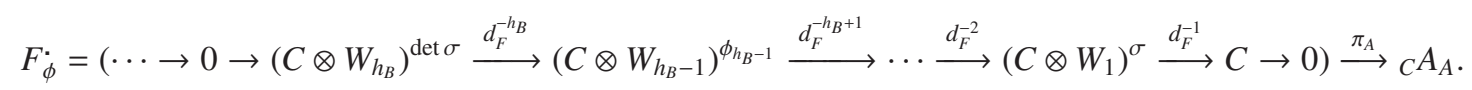

Obviously, there are two morphisms of cochain complexes:

$$
\iota_{0}: C \rightarrow F_{\dot{\phi}}^{\cdot}, \quad \pi_{h_{B}}: F_{\dot{\phi}}^{\cdot} \rightarrow\left(C \otimes W_{h_{B}}\right)^{\operatorname{det} \sigma}\left[h_{B}\right] .
$$

Lemma 3.5. Let $f \in V_{1}^{*}$ and $g \in W_{h_{B}}^{*}$. If $\sigma$ is invertible, then

$$
(1 \otimes f) \cdot(g \otimes 1)=g \otimes\left(f \circ(\operatorname{det} \sigma)_{\mid V_{1}}\right) \in W_{h_{B}}^{*} \otimes V_{1}^{*} \subseteq E^{h_{B}+1}(C) .
$$

Proof. Write $v=\operatorname{det} \sigma$. Let $\alpha: P \cdot \rightarrow{ }_{A} k(-1)[1]$ be the morphism of cochain complexes corresponding to $f$ and $\beta: Q \rightarrow{ }_{B} k\left(-l_{B}\right)\left[h_{B}\right]$ be the one corresponding to $g$. By Lemma 3.2 and Remark $3.1, E\left(\pi_{A}\right)(\alpha)$ : $F_{\phi} \otimes_{A} P^{\cdot} \rightarrow C^{k(-1)[1]}$ in $\mathrm{Ch}(\mathrm{Gr} C)$ corresponds to $1 \otimes f \in W_{0}^{*} \otimes V_{1}^{*}$ induced by

$$
\pi_{A} \otimes_{A}\left(\varepsilon_{A} \otimes f\right): C \otimes_{A}\left(A \otimes V_{1}\right) \rightarrow{ }_{C} k(-1),
$$

and $E\left(\pi_{B}\right)(\beta): F_{\dot{\phi}} \otimes_{A} P \cdot \rightarrow{ }_{C} k\left(-l_{B}\right)\left[h_{B}\right]$ in $\mathrm{Ch}(\mathrm{Gr} C)$ corresponds to $g \otimes 1 \in W_{h_{B}}^{*} \otimes V_{0}^{*}$ induced by

$$
\left(C \otimes W_{h_{B}}\right)^{v} \otimes_{A} A \cong C \otimes W_{h_{B}} \stackrel{\pi_{B} \otimes W_{h_{B}}}{\longrightarrow} B \otimes W_{h_{B}} \stackrel{\varepsilon_{B} \otimes g}{\longrightarrow} c k\left(-l_{B}\right) .
$$

Clearly, the cochain complex ${ }^{v^{-1}} P \cdot$ is also a minimal free resolution of ${ }_{A} k$ via quasi-isomorphism $\varepsilon_{A}$. For each $i$-th term of ${ }^{\nu^{-1}} P$, it is obvious ${ }^{\nu^{-1}}\left(A \otimes V_{i}\right) \cong\left({ }^{v^{-1}} A\right) \otimes V_{i}$ as graded $A$-modules. One can choose an isomorphism of cochain complexes $\rho:{ }^{v^{-1}} P^{\cdot} \rightarrow P^{*}$ in $\operatorname{Ch}(\operatorname{Gr} A)$ such that $\varepsilon_{A} \circ \rho=\varepsilon_{A}$ and

$$
\begin{array}{ll}
\rho^{0}=v: & v^{-1} A \rightarrow A, \\
\rho^{-1}=v \otimes v_{\mid V_{1}}: & \left({ }^{-1} A\right) \otimes V_{1} \rightarrow A \otimes V_{1} .
\end{array}
$$

The Yoneda product can be computed by the following commutative diagram in $\mathrm{Ch}(\mathrm{Gr} C)$ :

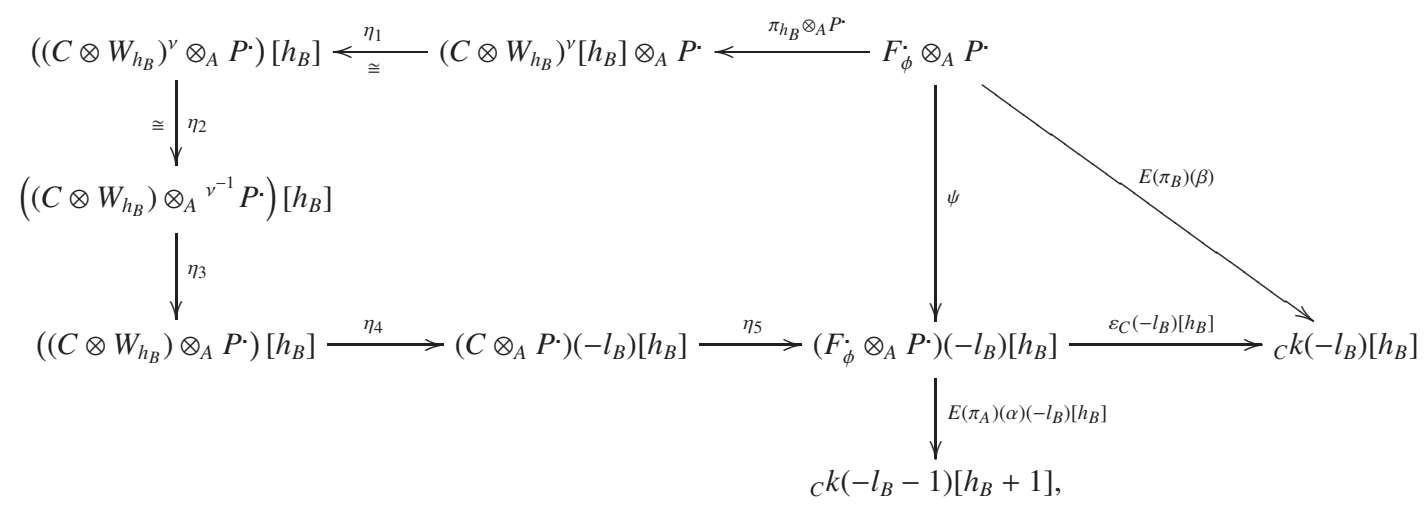


where $\psi:=\eta_{5} \circ \eta_{4} \circ \eta_{3} \circ \eta_{2} \circ \eta_{1} \circ\left(\pi_{h_{B}} \otimes_{A} P^{*}\right)$, the isomorphism $\eta_{1}$ is a natural shift of complexes without additional signs, the isomorphism $\eta_{2}$ acts on elements as the identity map, $\eta_{3}:=\left(\left(C \otimes W_{h_{B}}\right) \otimes_{A} \rho\right)\left[h_{B}\right]$, $\eta_{4}:=\left((C \otimes g) \otimes_{A} P^{\cdot}\right)\left[h_{B}\right]$, and $\eta_{5}:=\left(\iota \otimes_{A} P^{\cdot}\right)\left(-l_{B}\right)\left[h_{B}\right]$. It is easy to check that $\varepsilon_{C}\left(-l_{B}\right)\left[h_{B}\right] \circ \psi=E\left(\pi_{B}\right)(\beta)$.

Then $E\left(\pi_{A}\right)(\alpha) \circ E\left(\pi_{B}\right)(\beta)=E\left(\pi_{A}\right)(\alpha)\left(-l_{B}\right)\left[h_{B}\right] \circ \psi$, and the nonzero component equals composition of following morphisms in $\mathrm{Gr} C$

$\left(C \otimes W_{h_{B}}\right)^{v} \otimes_{A}\left(A \otimes V_{1}\right) \cong\left(C \otimes W_{h_{B}}\right) \otimes_{A}{ }^{v^{-1}}\left(A \otimes V_{1}\right) \stackrel{(C \otimes g) \otimes_{A}(v \otimes v)}{\longrightarrow} C \otimes_{A}\left(A \otimes V_{1}\right)\left(-l_{B}\right) \stackrel{\left(\pi_{A} \otimes_{A}\left(\varepsilon_{A} \otimes f\right)\right)\left(-l_{B}\right)}{\longrightarrow} C k\left(-l_{B}-1\right)$.

Hence $(1 \otimes f) \cdot(g \otimes 1)=g \otimes(f \circ v) \in W_{h_{B}}^{*} \otimes V_{1}^{*} \subseteq E^{h_{B}+1}(C)$ by Remark 3.1

3.3. When $A$ is AS-regular. In the final case, we assume $A$ is AS-regular of type $\left(h_{A}, l_{A}\right)$, and $\sigma$ is invertible. Write $\varphi=\sigma^{-1}$ and $U=k^{\oplus m}$ in the sequel.

The minimal resolution $(\underline{\mathrm{RA}})$ of ${ }_{A} k$ becomes:

$$
P^{*}:=\left(\cdots \rightarrow 0 \rightarrow A \otimes V_{h_{A}} \stackrel{d_{P}^{-h_{A}}}{\longrightarrow} A \otimes V_{h_{A}-1} \stackrel{d_{P}^{-h_{A}+1}}{\longrightarrow} \cdots \rightarrow A \otimes V_{1} \stackrel{d_{P}^{-1}}{\longrightarrow} A \rightarrow 0 \rightarrow \cdots\right) \stackrel{\varepsilon_{A}}{\longrightarrow} \quad{ }_{A} k,
$$

where $V_{h_{A}}=k\left(-l_{A}\right)$. Let $\omega$ be a basis of $V_{h_{A}}$. So the dual basis $\omega^{*}$ is a basis of $E^{h_{A}}(A)=V_{h_{A}}^{*}$.

By the definition, the homological determinant of $\sigma$ comes form the morphism $\theta:{ }^{\varphi}\left(P^{\cdot} \otimes U\right) \rightarrow P^{\cdot} \otimes U$ satisfying $\varepsilon_{A}^{\oplus m} \circ \theta=\varepsilon_{A}^{\oplus m}$. In the following, we use other morphisms to obtain homological determinant.

Let $\operatorname{pr}_{\mathrm{i}}:{ }_{A} k^{\oplus m} \rightarrow{ }_{A} k$ be the natural projection of $i$-th position for $1 \leq i \leq m$. Then there exists a morphism of cochain complexes in $\mathrm{Ch}(\mathrm{Gr} A)$

$$
\theta_{i}:{ }^{\varphi}(P \cdot \otimes U) \rightarrow P
$$

such that $\varepsilon_{A} \circ \theta_{i}=\operatorname{pr}_{i} \circ \varepsilon_{A}^{\oplus m}$ for $1 \leq i \leq m$.

Let $i$ be an arbitrary integer in the set $\{1, \cdots, m\}$. The action of functor $\underline{\operatorname{Hom}}_{A}(-, k)$ to $\theta_{i}$ produces a bigraded linear map $\underline{\operatorname{Hom}}_{A}\left(\theta_{i}, k\right): E(A) \rightarrow E(A)^{\oplus m}$. Restricted on $E^{h_{A}}(A)$, one obtains

$$
\underline{\operatorname{Hom}}_{A}\left(\theta_{i}, k\right)\left(\omega^{*}\right)=h_{i 1}^{\prime} \omega_{1}^{*}+h_{i 2}^{\prime} \omega_{2}^{*}+\cdots+h_{i m}^{\prime} \omega_{m}^{*},
$$

where $h_{i 1}^{\prime}, h_{i 2}^{\prime}, \cdots, h_{i m}^{\prime} \in k$ and $\left\{\omega_{i}^{*}\right\}$ is the canonical basis of $E^{h_{A}}(A)^{\oplus m}$ with respect to $\omega^{*}$. Now, we have another matrix $H^{\prime}=\left(h_{i j}^{\prime}\right) \in \mathbb{M}_{m}(k)$.

Lemma 3.6. The homological determinant hdet $\sigma=H^{\prime}$.

Proof. Let $i \in\{1, \cdots, m\}$. The natural projection $\mathrm{pr}_{i}: k^{\oplus m} \rightarrow k$ induces a natural projection $\widetilde{\mathrm{pr}}_{i}: P \cdot \otimes U \rightarrow$ $P$. satisfying $\varepsilon_{A} \circ \widetilde{\operatorname{pr}}_{i}=\mathrm{pr}_{i} \circ \varepsilon_{A}^{\oplus m}$. Now we consider the following commutative diagram

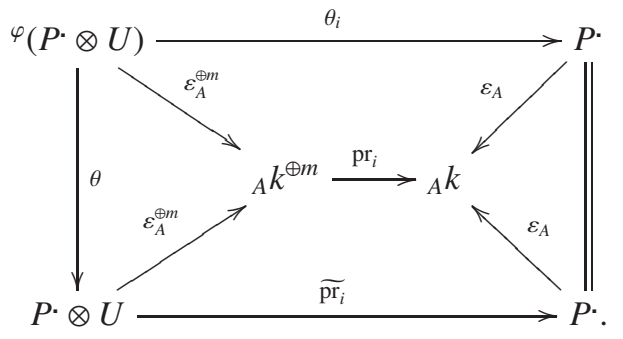

So $\varepsilon_{A} \circ \widetilde{\mathrm{pr}}_{i} \circ \theta=\varepsilon_{A} \circ \theta_{i}$. Because $\varepsilon_{A}: P \cdot \rightarrow{ }_{A} k$ is a quasi-isomorphism and $\underline{\operatorname{Hom}}_{A}\left({ }^{\varphi}\left(P^{\cdot} \otimes U\right),-\right)$ preserves quasi-isomorphism, $\widetilde{\mathrm{pr}}_{i} \circ \theta-\theta_{i}$ is null homotopic. That is to say

$$
\underline{\operatorname{Hom}}_{A}\left(\theta_{i}, k\right)=\underline{\operatorname{Hom}}_{A}\left(\widetilde{\mathrm{pr}}_{i} \circ \theta, k\right): E(A) \rightarrow E(A)^{\oplus m} .
$$


However, it is obvious $\underline{\operatorname{Hom}}_{A}\left(\widetilde{\mathrm{pr}}_{i}, k\right): E(A) \rightarrow E(A)^{\oplus m}$ is a natural injection of $i$-th position. Hence,

$$
\underline{\operatorname{Hom}}_{A}\left(\theta_{i}, k\right)\left(\omega^{*}\right)=\underline{\operatorname{Hom}}_{A}(\theta, k) \circ \underline{\operatorname{Hom}}_{A}\left(\widetilde{\mathrm{pr}}_{i}, k\right)\left(\omega^{*}\right)=\underline{\operatorname{Hom}}_{A}(\theta, k)\left(\omega_{i}^{*}\right) .
$$

The proof is completed.

Recall that the minimal free resolution $(\underline{\mathrm{RB}})$ of ${ }_{B} k$ is

$$
Q=\left(\cdots \stackrel{d_{Q}^{-4}}{\longrightarrow} B \otimes W_{3} \stackrel{d_{Q}^{-3}}{\longrightarrow} B \otimes W_{2} \stackrel{d_{Q}^{-2}}{\longrightarrow} B \otimes W_{1} \stackrel{d_{Q}^{-1}}{\longrightarrow} B \rightarrow 0 \rightarrow \cdots\right) \stackrel{\varepsilon_{B}}{\longrightarrow} \quad{ }_{B} k,
$$

where $W_{1}=k\left(-l_{1}\right)^{\oplus m}$ with the canonical coordinate basis $\left\{e_{1}, e_{2}, \cdots, e_{m}\right\}$. The dual basis $\left\{e_{1}^{*}, e_{2}^{*}, \cdots, e_{m}^{*}\right\}$ of $W_{1}^{*}$ can be seen as the basis elements of $E^{1}(B)$.

It is easy to know that graded $A$-module homomorphism $A \otimes e_{i}^{*}: A \otimes W_{1} \rightarrow A\left(-l_{1}\right)$ is also a graded right $A$-module homomorphism. Then we have a graded $A^{e}$-module homomorphism

$$
\Psi:=\left(A \otimes e_{1}^{*}, \cdots, A \otimes e_{m}^{*}\right):\left(A \otimes W_{1}\right)^{\sigma} \rightarrow\left(A\left(-l_{1}\right)^{\oplus m}\right)^{\sigma} \cong(A \otimes U)^{\sigma}\left(-l_{1}\right) .
$$

Lemma 3.7. For any graded A-module $M$, there exists a natural isomorphism as graded A-modules

$$
(A \otimes U)^{\sigma} \otimes_{A} M \cong{ }^{\varphi}(M \otimes U) .
$$

Proof. Let $\left\{u_{1}, u_{2}, \cdots, u_{m}\right\}$ be the canonical coordinate basis of $U$. Define

$$
\begin{aligned}
& \zeta: \quad(A \otimes U)^{\sigma} \otimes_{A} M \quad \rightarrow \quad A \otimes_{A} \varphi(M \otimes U) \stackrel{\cong}{\rightarrow} \varphi(M \otimes U) \\
& \left(\sum_{i=1}^{m} a_{i} \otimes u_{i}\right) \otimes_{A} x \quad \mapsto \quad \sum_{i=1}^{m} a_{i} \otimes_{A}\left(x \otimes u_{i}\right) \quad \mapsto \quad \sum_{i=1}^{m}\left(\sum_{j=1}^{m}\left(\varphi_{j i}\left(a_{i}\right) x \otimes u_{j}\right) .\right.
\end{aligned}
$$

It is easy to check it is a natural graded $A$-module isomorphism.

Using the isomorphism in the lemma above, one obtains a morphism of cochain complexes in $\mathrm{Ch}(\mathrm{Gr} A)$ :

$$
\xi_{i}:\left(A \otimes W_{1}\right)^{\sigma} \otimes_{A} P \cdot \stackrel{\Psi \otimes_{A} P \cdot}{\longrightarrow}(A \otimes U)^{\sigma}\left(-l_{1}\right) \otimes_{A} P \cdot \stackrel{\cong}{\longrightarrow} \varphi(P \cdot \otimes U)\left(-l_{1}\right) \stackrel{\theta_{i}\left(-l_{1}\right)}{\longrightarrow} P^{\cdot}\left(-l_{1}\right),
$$

where $i=1, \cdots, m$. By Lemma 3.6 and Lemma3.7, we have an immediate result.

Lemma 3.8. $\left(\left(\xi_{1}\right)^{-h_{A}}, \cdots,\left(\xi_{m}\right)^{-h_{A}}\right)^{T}=\operatorname{hdet} \sigma \cdot\left(\left(A \otimes e_{1}^{*}\right) \otimes_{A} A\left(-l_{A}\right), \cdots,\left(A \otimes e_{m}^{*}\right) \otimes_{A} A\left(-l_{A}\right)\right)^{T}$.

Now it is the turn to show a result about the Yoneda product.

Lemma 3.9. Let $f \in V_{h^{A}}^{*}$, then

$$
(1 \otimes f) \cdot\left(e_{1}^{*} \otimes 1, \cdots, e_{m}^{*} \otimes 1\right)^{T}=\operatorname{hdet} \sigma \cdot\left(e_{1}^{*} \otimes f, \cdots, e_{m}^{*} \otimes f\right)^{T} .
$$

Proof. Write $\alpha: P \cdot \rightarrow{ }_{A} k\left(-l_{A}\right)\left[h_{A}\right]$ to be the morphism of cochain complexes corresponding to $f$, and $\beta_{i}: Q \rightarrow{ }_{B} k\left(-l_{1}\right)[1]$ to be the one corresponding to $e_{i}^{*}$ for $i=1, \cdots, m$. By Lemma 3.2 and Remark 3.1, $E\left(\pi_{A}\right)(\alpha): F_{\dot{\phi}} \otimes_{A} P^{\cdot} \rightarrow{ }_{C} k\left(-l_{A}\right)\left[h_{A}\right]$ in $\mathrm{Ch}(\mathrm{Gr} C)$ corresponds to $1 \otimes f \in W_{0}^{*} \otimes V_{h_{A}}^{*}$ induced by

$$
\pi_{A} \otimes_{A}\left(\varepsilon_{A} \otimes f\right): C \otimes_{A}\left(A \otimes V_{h_{A}}\right) \rightarrow{ }_{C} k\left(-l_{A}\right),
$$

and each $E\left(\pi_{B}\right)\left(\beta_{i}\right): F_{\dot{\phi}} \otimes_{A} P^{\cdot} \rightarrow{ }_{C} k\left(-l_{1}\right)[1]$ in $\mathrm{Ch}(\mathrm{Gr} C)$ corresponds to $e_{i}^{*} \otimes 1 \in W_{1}^{*} \otimes V_{0}^{*}$ induced by

$$
\left(C \otimes W_{1}\right)^{\sigma} \otimes_{A} A \cong C \otimes W_{1} \stackrel{\pi_{B} \otimes W_{1}}{\longrightarrow} B \otimes W_{1} \stackrel{\varepsilon_{B} \otimes e_{i}^{*}}{\longrightarrow} C k\left(-l_{1}\right) .
$$

The graded $(C, A)$-bimodule homomorphism $\pi_{A} \otimes W_{1}:\left(C \otimes W_{1}\right)^{\sigma} \rightarrow\left(A \otimes W_{1}\right)^{\sigma}$ induces a morphism of cochain complexes in $\mathrm{Ch}\left(\mathrm{Gr}\left(C \otimes A^{\circ}\right)\right)$

$$
\eta: F_{\dot{\phi}} \rightarrow\left(A \otimes W_{1}\right)^{\sigma}[1]
$$


since the differential $d_{F}=A \otimes^{\tau} d_{Q}$ and $Q \cdot$ is minimal.

Let $i$ be an arbitrary integer. Then we focus on the following diagram

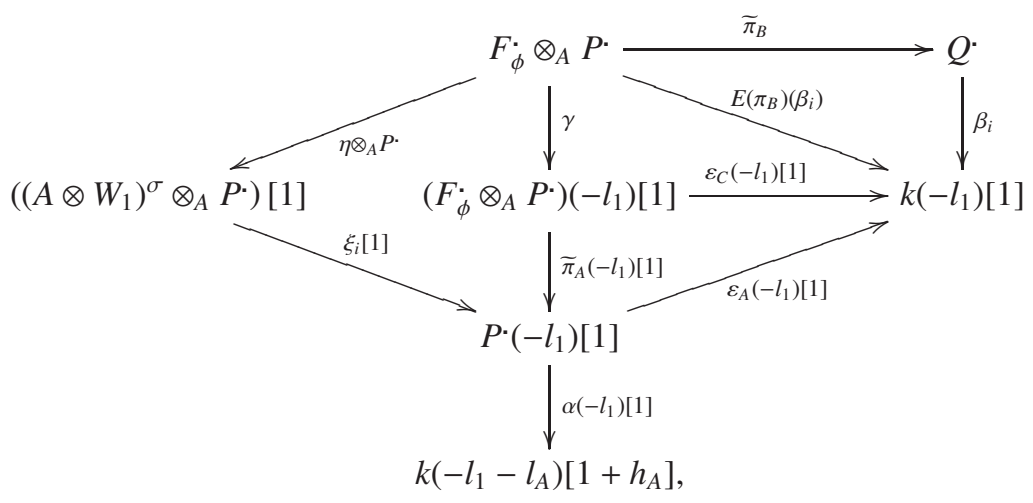

where $\gamma$ is chosen to satisfy $\varepsilon_{C}\left(-l_{1}\right)[1] \circ \gamma=E\left(\pi_{B}\right)\left(\beta_{i}\right)=\beta_{i} \circ \widetilde{\pi}_{B}$. It is clear the right half of the diagram above is commutative, so $\varepsilon_{A}\left(-l_{1}\right)[1] \circ \widetilde{\pi}_{A}\left(-l_{1}\right)[1] \circ \gamma=\beta_{i} \circ \widetilde{\pi}_{B}$. By a straightforward computation, one obtains $\varepsilon_{A}\left(-l_{1}\right)[1] \circ \xi_{i}[1] \circ\left(\eta \otimes_{A} P^{\cdot}\right)=\beta_{i} \circ \widetilde{\pi}_{B}$, that is,

$$
\varepsilon_{A}\left(-l_{1}\right)[1] \circ \widetilde{\pi_{A}}\left(-l_{1}\right)[1] \circ \gamma=\varepsilon_{A}\left(-l_{1}\right)[1] \circ \xi_{i}[1] \circ\left(\eta \otimes_{A} P^{\cdot}\right) .
$$

Because $\varepsilon_{A}$ is a quasi-isomorphism, $\widetilde{\pi_{A}}\left(-l_{1}\right)[1] \circ \gamma$ is homotopic to $\xi_{i}[1] \circ\left(\eta \otimes_{A} P \cdot\right)$. Combined with the minimality of resolutions $F_{\dot{\phi}}$ and $P$, we have

$$
\alpha\left(-l_{1}\right)[1] \circ \widetilde{\pi_{A}}\left(-l_{1}\right)[1] \circ \gamma=\alpha\left(-l_{1}\right)[1] \circ \xi_{i}[1] \circ\left(\eta \otimes_{A} P^{*}\right) .
$$

In the other hand, $E\left(\pi_{A}\right)(\alpha) \cdot E\left(\pi_{B}\right)\left(\beta_{i}\right)=\alpha\left(-l_{1}\right)[1] \circ \widetilde{\pi}_{A}\left(-l_{1}\right)[1] \circ \gamma=\alpha\left(-l_{1}\right)[1] \circ \xi_{i}[1] \circ\left(\eta \otimes_{A} P \cdot\right)$ and the nonzero component equals the composition of following morphisms

$\left(C \otimes W_{1}\right)^{\sigma} \otimes_{A}\left(A \otimes V_{h_{A}}\right) \stackrel{\left(\pi_{A} \otimes W_{1}\right) \otimes_{A}\left(A \otimes V_{h_{A}}\right)}{\longrightarrow}\left(A \otimes W_{1}\right)^{\sigma} \otimes_{A}\left(A \otimes V_{h_{A}}\right) \stackrel{\left(\xi_{i}\right)^{-h_{A}}}{\longrightarrow}\left(A \otimes V_{h_{A}}\right)\left(-l_{1}\right) \stackrel{\left(\varepsilon_{A} \otimes f\right)\left(-l_{1}\right)}{\longrightarrow} C k\left(-l_{1}-l_{A}\right)$.

Hence the result follows by Lemma 3.8 and Remark 3.1

\section{Nakayama Automorphisms}

With the preparations of results for Ext-algebras, we turn to describe Nakayama automorphisms of twisted tensor products. We still keep to the Hypothesis 2.6 firstly. In addition, we assume connected graded algebras $A$ and $B$ are noetherian AS-regular algebras of type $\left(h_{A}, l_{A}\right)$ and $\left(h_{B}, l_{B}\right)$ respectively which are both generated in degree 1 , and the graded algebra homomorphism $\sigma$ is invertible.

By Theorem 2.11, $A \otimes^{\tau} B$ is AS-regular and has Nakayama automorphism. We study the Nakayama automorphism of $A \otimes^{\tau} B$ by taking advantage of the classical Nakayama automorphism of Ext-algebra $E\left(A \otimes^{\tau} B\right)$. It depends on the Yoneda product of $E\left(A \otimes^{\tau} B\right) \cong E(B) \otimes^{\tau_{E}} E(A)$ for the bigraded twisting map $\tau_{E}$ occurred in Theorem 3.4 The last two cases of Section 3 make us be able to describe the bigraded linear map $\tau_{E}$ restricted on $E^{1}(A) \otimes E^{h_{B}}(B)$ and $E^{h_{A}}(A) \otimes E^{1}(B)$. Fortunately, it suffices to realize the goal for Nakayama automorphisms.

Under the assumption, $E^{1}(A)=A_{1}^{*}$ and $E^{1}(B)=B_{1}^{*}$. The $k$-dual of determinant $(\operatorname{det} \sigma)^{*}$ is an automorphism of $E^{1}(A)$, and $E^{1}(B)$ has a basis $\left\{y_{1}^{*}, \cdots, y_{m}^{*}\right\}$. 
Lemma 4.1. Let $f_{1} \in E^{1}(A), f_{h_{A}} \in E^{h_{A}}(A)$ and $g \in E^{h_{B}}(B)$, then

$$
\begin{aligned}
& \tau_{E}\left(f_{1} \otimes g\right)=(-1)^{h_{B}} g \otimes\left(\operatorname{det} \sigma_{\mid A_{1}}\right)^{*}\left(f_{1}\right), \\
& \tau_{E}\left(\begin{array}{c}
f_{h_{A}} \otimes y_{1}^{*} \\
f_{h_{A}} \otimes y_{2}^{*} \\
\vdots \\
f_{h_{A}} \otimes y_{m}^{*}
\end{array}\right)=(-1)^{h_{A}} \operatorname{hdet} \sigma \cdot\left(\begin{array}{c}
y_{1}^{*} \otimes f_{h_{A}} \\
y_{2}^{*} \otimes f_{h_{A}} \\
\vdots \\
y_{m}^{*} \otimes f_{h_{A}}
\end{array}\right) .
\end{aligned}
$$

Proof. Write $m_{E}$ to be the Yoneda product of the Ext-algebra $E\left(A \otimes^{\tau} B\right)$. By Lemma3.5, we have

$$
m_{E} \circ\left(E\left(\pi_{A}\right) \otimes E\left(\pi_{B}\right)\right)\left(f_{1} \otimes g\right)=\left(1 \otimes f_{1}\right) \cdot(g \otimes 1)=g \otimes\left(\operatorname{det} \sigma_{\mid A_{1}}\right)^{*}\left(f_{1}\right) \in E\left(A \otimes^{\tau} B\right) .
$$

In the other hand, following from Lemma 3.3 , one obtains

$$
\begin{aligned}
m_{E} \circ\left(E\left(\pi_{B}\right) \otimes E\left(\pi_{A}\right)\right)\left(g \otimes\left(\operatorname{det} \sigma_{\mid A_{1}}\right)^{*}\left(f_{1}\right)\right) & =(g \otimes 1) \cdot\left(1 \otimes\left(\operatorname{det} \sigma_{\mid A_{1}}\right)^{*}\left(f_{1}\right)\right) \\
& =(-1)^{h_{B}} g \otimes\left(\operatorname{det} \sigma_{\mid A_{1}}\right)^{*}\left(f_{1}\right) \in E\left(A \otimes^{\tau} B\right) .
\end{aligned}
$$

Hence, we have

$$
\tau_{E}\left(f_{1} \otimes g\right)=\left(m_{E} \circ\left(E\left(\pi_{B}\right) \otimes E\left(\pi_{A}\right)\right)\right)^{-1} \circ m_{E} \circ\left(E\left(\pi_{A}\right) \otimes E\left(\pi_{B}\right)\right)\left(f_{1} \otimes g\right)=(-1)^{h_{B}} g \otimes\left(\operatorname{det} \sigma_{\mid A_{1}}\right)^{*}\left(f_{1}\right),
$$

by Theorem 3.4. The second equation follows from Lemma 3.9 similarly.

Theorem 4.2. Let $A=k\langle X\rangle /\left(G_{A}\right)$ and $B=k\langle Y\rangle /\left(G_{B}\right)$ be both noetherian AS-regular algebras generated in degree 1, where $X=\left\{x_{i}\right\}_{i=1}^{n}$ and $Y=\left\{y_{j}\right\}_{j=1}^{m}$ are minimal generating sets of $A$ and $B$ respectively. Let $\tau=(\sigma, 0): B \otimes A \rightarrow A \otimes B$ be a graded twisting map such that $A \otimes^{\tau} B$ is noetherian. Suppose $B$ has a pure resolution and $\sigma$ is invertible, then the Nakayama automorphism $\mu_{A \otimes^{\top} B}$ satisfies

$$
\begin{aligned}
& \mu_{A \otimes^{\top} B \mid A}=(\operatorname{det} \sigma)^{-1} \circ \mu_{A}, \\
& \mu_{A \otimes^{\top} B \mid B}\left(\begin{array}{c}
y_{1} \\
y_{2} \\
\vdots \\
y_{m}
\end{array}\right)=\operatorname{hdet} \sigma \cdot \mu_{B}\left(\begin{array}{c}
y_{1} \\
y_{2} \\
\vdots \\
y_{m}
\end{array}\right) .
\end{aligned}
$$

Proof. Write $C=A \otimes^{\tau} B$. Denote the Ext-algebras of $A, B$ and $C$ by $E(A), E(B)$ and $E(C)$ respectively. Let $\left\{x_{i}^{*}\right\}_{i=1}^{n}$ and $\left\{y_{j}^{*}\right\}_{j=1}^{m}$ be dual base of $E^{1}(A)$ and $E^{1}(B)$ respectively. Write $v=\operatorname{det} \sigma$.

Assume $A$ and $B$ are AS-regular algebras of type $\left(h_{A}, l_{A}\right)$ and $\left(h_{B}, l_{B}\right)$ respectively. By Theorem 2.11 $C$ is AS-regular of type $\left(h_{A}+h_{B}, l_{A}+l_{B}\right)$. Following from Theorem 1.4 a), $E(A), E(B)$ and $E(C)$ are Frobenius algebras of type $\left(-h_{A}, l_{A}\right),\left(-h_{B}, l_{B}\right)$ and $\left(-h_{A}-h_{B}, l_{A}+l_{B}\right)$ respectively. Denote the (classical) Nakayama automorphisms of $E(A), E(B)$ and $E(C)$ by $\mu_{E(A)}, \mu_{E(B)}$ and $\mu_{E(C)}$ respectively.

By Theorem 3.4, there exists a bigraded linear map $\tau_{E}: E(A) \otimes E(B) \rightarrow E(B) \otimes E(A)$ such that $E(C)=$ $E(B) \otimes^{\tau_{E}} E(A)$. Since $A$ and $B$ are generated in degree $1,\left\{1 \otimes^{\tau_{E}} x_{1}^{*}, \cdots, 1 \otimes^{\tau_{E}} x_{n}^{*}, y_{1}^{*} \otimes^{\tau_{E}} 1, \cdots, y_{m}^{*} \otimes^{\tau_{E}} 1\right\}$ is a basis of $E^{1}(C)$.

Firstly, we focus on $\mu_{E(C)}\left(1 \otimes^{\tau_{E}} x_{i}^{*}\right)$. Let $\omega_{B}$ be a basis of $E^{h_{B}}(B)$, and $\left\{f_{1}, \cdots, f_{n}\right\}$ be a basis of $E^{h_{A}-1}(A)$. Since the degree of bigraded linear form for $E(C)$ is $\left(-h_{A}-h_{B}, l_{A}+l_{B}\right)$, the only possible nonzero values come from

$$
\left\langle 1 \otimes^{\tau_{E}}\left(v^{-1}\right)^{*}\left(x_{i}^{*}\right), \omega_{B} \otimes^{\tau_{E}} f_{j}\right\rangle=\left\langle\left(1 \otimes^{\tau_{E}}\left(v^{-1}\right)^{*}\left(x_{i}^{*}\right)\right)\left(\omega_{B} \otimes^{\tau_{E}} 1\right), 1 \otimes^{\tau_{E}} f_{j}\right\rangle,
$$


following from the associativity of bilinear form for any $i, j=1, \cdots, n$. By Lemma 4.1 , we have

$$
\left(1 \otimes^{\tau_{E}}\left(v^{-1}\right)^{*}\left(x_{i}^{*}\right)\right)\left(\omega_{B} \otimes^{\tau_{E}} 1\right)=\omega_{B} \otimes^{\tau_{E}}(-1)^{h_{B}} x_{i}^{*} .
$$

Using the associativity again,

$$
\begin{aligned}
\left\langle 1 \otimes^{\tau_{E}}\left(v^{-1}\right)^{*}\left(x_{i}^{*}\right), \omega_{B} \otimes^{\tau_{E}} f j\right\rangle & =\left\langle\omega_{B} \otimes^{\tau_{E}}(-1)^{h_{B}} x_{i}^{*}, 1 \otimes^{\tau_{E}} f_{j}\right\rangle \\
& =(-1)^{h_{B}}\left\langle\omega_{B} \otimes^{\tau_{E}} 1,1 \otimes^{\tau_{E}} x_{i}^{*} f_{j}\right\rangle \\
& =(-1)^{h_{B}+h_{A}-1}\left\langle\omega_{B} \otimes^{\tau_{E}} 1,1 \otimes^{\tau_{E}} f_{j} \mu_{E(A)}^{-1}\left(x_{i}^{*}\right)\right\rangle \\
& =(-1)^{h_{B}+h_{A}-1}\left\langle\omega_{B} \otimes^{\tau_{E}} f_{j}, 1 \otimes^{\tau_{E}} \mu_{E(A)}^{-1}\left(x_{i}^{*}\right)\right\rangle .
\end{aligned}
$$

So $\mu_{E(C)}\left(1 \otimes^{\tau_{E}} x_{i}^{*}\right)=1 \otimes^{\tau_{E}}\left(v^{-1}\right)^{*} \mu_{E(A)}\left(x_{i}^{*}\right)$. It implies

$$
\mu_{E(C) \mid E^{1}(A)}=\left.\left(v^{-1}\right)^{*} \mu_{E(A)}\right|_{E^{1}(A)} .
$$

By Theorem 1.4 (b) and the noetherianess of $C$,

$$
\mu_{C \mid A_{1}}=\left(\mu_{E(C) \mid E^{1}(A)}\right)^{*}=\left(\left(v^{-1}\right)^{*} \circ \mu_{E(A)}\right)^{*}=\mu_{E(A)}^{*} \circ v^{-1}=\mu_{A} \circ v^{-1}{ }_{\mid A_{1}} .
$$

In conclusion, $\mu_{C \mid A}=\mu_{A} \circ(\operatorname{det} \sigma)^{-1}=(\operatorname{det} \sigma)^{-1} \circ \mu_{A}$, since the Nakayama automorphism is in the center of automorphism group of algebra by [9, Theorem 0.6].

Now we turn to consider $\mu_{E(C)}\left(y_{j}^{*} \otimes^{\tau_{E}} 1\right)$. Let $\omega_{A}$ be a basis of $E^{h_{A}}(A)$, and $\left\{g_{1}, \cdots, g_{m}\right\}$ be a basis of $E^{h_{B}-1}(B)$. Using the associativity of bilinear form $\langle\cdot, \cdot\rangle$ of $E(C)$, we have the only possible nonzero values

$$
\left\langle g_{i} \otimes^{\tau_{E}} \omega_{A}, y_{j}^{*} \otimes^{\tau_{E}} 1\right\rangle=\left\langle g_{i} \otimes^{\tau_{E}} 1,\left(1 \otimes^{\tau_{E}} \omega_{A}\right)\left(y_{j}^{*} \otimes^{\tau_{E}} 1\right)\right\rangle,
$$

for any $i, j=1,2, \cdots, m$. By Lemma 4.1

$$
\left(1 \otimes^{\tau_{E}} \omega_{A}\right)\left(y_{1}^{*} \otimes^{\tau_{E}} 1, \cdots, y_{m}^{*} \otimes^{\tau_{E}} 1\right)^{T}=(-1)^{h_{A}} \operatorname{hdet} \sigma \cdot\left(y_{1}^{*} \otimes^{\tau_{E}} \omega_{A}, \cdots, y_{m}^{*} \otimes^{\tau_{E}} \omega_{A}\right)^{T} .
$$

By the associativity again,

$$
\begin{aligned}
& \left(\left\langle g_{i} \otimes^{\tau_{E}} \omega_{A}, y_{1}^{*} \otimes^{\tau_{E}} 1\right\rangle, \cdots,\left\langle g_{i} \otimes^{\tau_{E}} \omega_{A}, y_{m}^{*} \otimes^{\tau_{E}} 1\right\rangle\right)^{T} \\
= & (-1)^{h_{A}} \operatorname{hdet} \sigma \cdot\left(\left\langle g_{i} \otimes^{\tau_{E}} 1, y_{1}^{*} \otimes^{\tau_{E}} \omega_{A}\right\rangle, \cdots,\left\langle g_{i} \otimes^{\tau_{E}} 1, y_{m}^{*} \otimes^{\tau_{E}} \omega_{A}\right\rangle\right)^{T} \\
= & (-1)^{h_{A}} \operatorname{hdet} \sigma \cdot\left(\left\langle g_{i} y_{1}^{*} \otimes^{\tau_{E}} 1,1 \otimes^{\tau_{E}} \omega_{A}\right\rangle, \cdots,\left\langle g_{i} y_{m}^{*} \otimes^{\tau_{E}} 1,1 \otimes^{\tau_{E}} \omega_{A}\right\rangle\right)^{T} \\
= & (-1)^{h_{A}+h_{B}-1} \operatorname{hdet} \sigma \cdot\left(\left\langle\mu_{E(B)}\left(y_{1}^{*}\right) g_{i} \otimes^{\tau_{E}} 1,1 \otimes^{\tau_{E}} \omega_{A}\right\rangle, \cdots,\left\langle\mu_{E(B)}\left(y_{m}^{*}\right) g_{i} \otimes^{\tau_{E}} 1,1 \otimes^{\tau_{E}} \omega_{A}\right\rangle\right)^{T} \\
= & (-1)^{h_{A}+h_{B}-1} \operatorname{hdet} \sigma \cdot\left(\left\langle\mu_{E(B)}\left(y_{1}^{*}\right) \otimes^{\tau_{E}} 1, g_{i} \otimes^{\tau_{E}} \omega_{A}\right\rangle, \cdots,\left\langle\mu_{E(B)}\left(y_{m}^{*}\right) \otimes^{\tau_{E}} 1, g_{i} \otimes^{\tau_{E}} \omega_{A}\right\rangle\right)^{T} .
\end{aligned}
$$

Thus $\left(\mu_{E(C)}\left(y_{1}^{*} \otimes^{\tau_{E}} 1\right), \cdots, \mu_{E(C)}\left(y_{m}^{*} \otimes^{\tau_{E}} 1\right)\right)^{T}=\operatorname{hdet} \sigma \cdot\left(\mu_{E(B)}\left(y_{1}^{*}\right) \otimes^{\tau_{E}} 1, \cdots, \mu_{E(B)}\left(y_{m}^{*}\right) \otimes^{\tau_{E}} 1\right)^{T}$, and

$$
\mu_{E(C) \mid E^{1}(B)}\left(y_{1}^{*}, \cdots, y_{m}^{*}\right)^{T}=\operatorname{hdet} \sigma \cdot \mu_{E(B)}\left(y_{1}^{*}, \cdots, y_{m}^{*}\right)^{T} .
$$

By Theorem 1.4 b), the noetherianess of $C$ and Proposition 1.10 ,

$$
\mu_{C \mid B}\left(\begin{array}{c}
y_{1} \\
y_{2} \\
\vdots \\
y_{m}
\end{array}\right)=\operatorname{hdet} \sigma \cdot \mu_{B}\left(\begin{array}{c}
y_{1} \\
y_{2} \\
\vdots \\
y_{m}
\end{array}\right) .
$$

The proof is completed. 
Finally, we assume the graded twisting map $\tau=(\sigma, \delta): B \otimes A \rightarrow A \otimes B$ with nonzero $\sigma$-derivation $\delta$. In this case, the related graded linear map $\bar{\tau}=(\sigma, 0): B \otimes A \rightarrow A \otimes B$ with zero $\sigma$-derivation is also a graded twisting map. We link the two twisted tensor products by a filtration of $A \otimes^{\tau} B$.

For any monomial $w$ in $k\langle X\rangle$ and $k\langle Y\rangle$, we have a canonical definition of length for $w$, denoted by $l(w)$. Give a monid homomorphism $l^{\prime}$ from $k\langle X, Y\rangle$ to $\mathbb{N}$ satisfying

$$
l^{\prime}(x)=l(x) \quad \text { and } \quad l^{\prime}(y)=l(y)+1,
$$

where $x \in X$ and $y \in Y$. So there is a filtration on $k\langle X, Y\rangle$ such that $F_{i}(k\langle X, Y\rangle)$ is a vector space spanned by monomials $w$ in $k\langle X, Y\rangle$ with $l^{\prime}(w) \leq i$ for $i \in \mathbb{Z}$. Since $A \otimes^{\tau} B \cong k\langle X, Y\rangle / I$ for some ideal $I$ of $k\langle X, Y\rangle$, there is a natural filtration

$$
F_{i}\left(A \otimes^{\tau} B\right):=\frac{F_{i}(k\langle X, Y\rangle)+I}{I}, \quad \forall i \in \mathbb{Z} .
$$

The associated graded algebra $\operatorname{gr}\left(A \otimes^{\tau} B\right) \cong A \otimes^{\bar{\tau}} B$ as graded algebras. We say $A \otimes^{\bar{\tau}} B$ is the associated twisted tensor product of $A \otimes^{\tau} B$.

Theorem 4.3. Let $A=k\langle X\rangle /\left(G_{A}\right)$ and $B=k\langle Y\rangle /\left(G_{B}\right)$ be both noetherian AS-regular algebras generated in degree 1 , where $Y=\left\{y_{i}\right\}_{i=1}^{m}$ is the minimal generating set of $B$. Let $\tau=(\sigma, \delta): B \otimes A \rightarrow A \otimes B$ be a graded twisting map such that the associated twisted tensor product $A \otimes^{\overline{ }} B$ is noetherian. Suppose $B$ has a pure resolution and $\sigma$ is invertible, then Nakayama automorphism $\mu_{A \otimes^{\top} B}$ satisfies

$$
\begin{aligned}
& \mu_{A \otimes^{\tau} B \mid A}=(\operatorname{det} \sigma)^{-1} \circ \mu_{A}, \\
& \mu_{A \otimes^{\tau} B}\left(\begin{array}{c}
1 \otimes^{\tau} y_{1} \\
1 \otimes^{\tau} y_{2} \\
\vdots \\
1 \otimes^{\tau} y_{m}
\end{array}\right)=\operatorname{hdet} \sigma \cdot\left(A \otimes^{\tau} \mu_{B}\right)\left(\begin{array}{c}
1 \otimes^{\tau} y_{1} \\
1 \otimes^{\tau} y_{2} \\
\vdots \\
1 \otimes^{\tau} y_{m}
\end{array}\right)+\left(\begin{array}{c}
a_{1} \otimes^{\tau} 1 \\
a_{2} \otimes^{\tau} 1 \\
\vdots \\
a_{m} \otimes^{\tau} 1
\end{array}\right),
\end{aligned}
$$

for some $a_{1}, a_{2}, \cdots, a_{m} \in A$.

Proof. By the discussion above this theorem, there is a filtration of $A \otimes^{\tau} B$ such that the associated graded algebra $\operatorname{gr}\left(A \otimes^{\tau} B\right) \cong A \otimes^{\bar{\tau}} B$ where $\bar{\tau}=(\sigma, 0)$. The Nakayama automorphism $\mu_{A \otimes^{\tau} B}$ of $A \otimes^{\tau} B$ is filtered and gr $\mu_{A \otimes^{\tau} B}=\mu_{A \otimes^{\tau} B}$ by [16, Lemma 5]. The result follows from Theorem 4.2] and the construction of filtration.

Remark 4.4. From Proposition 2.10, it is easy to know the twisted tensor product of Koszul algebras is also Koszul. If the noetherian condition is replaced by Koszul, the Theorem 1.4 b) still hold by [2, Theorem 1.3]. So we can release the noetherian conditions in Theorem 4.2 and 4.3 if the connected graded algebras $A$ and $B$ are Koszul algebras.

\section{EXAMPLES}

We conclude this paper with some applications of Theorem 4.3 .

Example 5.1. The classical Ore extension $A[z ; \sigma, \delta]$ is a twisted tensor product $A \otimes^{\tau} k[z]$ with

$$
\tau(z \otimes a)=\sigma(a) \otimes z+\delta(a) \otimes 1, \quad \forall a \in A,
$$

that is, $\tau=(\sigma, \delta)$. In this case, $\operatorname{det} \sigma=\sigma$ and $\operatorname{hdet} \sigma$ is just the one defined in [6]. The Nakayama of polynomial algebra $k[z]$ is the identity map. If $A$ is a noetherian AS-regular algebra with Nakayama 
automorphism $\mu_{A}$ generated in degree 1 and $\sigma$ is an automorphism of $A$, then the Nakayama automorphism $\mu$ of $A[z ; \sigma, \delta]$ satisfies

$$
\begin{aligned}
& \mu(a)=\sigma^{-1} \circ \mu_{A}(a), \quad \forall a \in A \\
& \mu(z)=(\operatorname{hdet} \sigma) z+a^{\prime},
\end{aligned}
$$

for some $a^{\prime} \in A$, by Theorem 4.3. It coincides with the result [7, Theorem 2].

Example 5.2. The second example is double Ore extension with zero tails $A_{P}\left[y_{1}, y_{2} ; \sigma, \delta\right]$, where $P=$ $\left\{p_{12}, p_{11}\right\} \subseteq k$, algebra homomorphism $\sigma: A \rightarrow \mathbb{M}_{2}(A)$ and $\sigma$-derivation $\delta: A \rightarrow A^{\oplus 2}$ (see [20] for details). It is a twisted tensor product of $A$ and $B=k\left\langle y_{1}, y_{2}\right\rangle /\left(y_{2} y_{1}-p_{12} y_{1} y_{2}-p_{11} y_{1}^{2}\right)$ with

$$
\tau\left(a \otimes y_{i}\right)=\sigma_{i 1}(a) \otimes y_{1}+\sigma_{i 2}(a) \otimes y_{2}+\delta_{i}(a) \otimes 1, \quad \forall a \in A, i=1,2 .
$$

So $\tau=(\sigma, \delta)$, and $\operatorname{det} \sigma=-p_{11} \sigma_{12} \sigma_{11}+\sigma_{22} \sigma_{11}-p_{12} \sigma_{12} \sigma_{21}$. If $p_{12}$ is nonzero, $B$ is Koszul AS-regular with Nakayama automorphism $\mu_{B}$ :

$$
\mu_{B}\left(\begin{array}{l}
y_{1} \\
y_{2}
\end{array}\right)=\left(\begin{array}{cc}
p_{12}^{-1} & 0 \\
p_{11}\left(1+p_{12}^{-1}\right) & p_{12}
\end{array}\right)\left(\begin{array}{l}
y_{1} \\
y_{2}
\end{array}\right)
$$

and $\operatorname{det} \sigma$ also equals $-p_{12}^{-1} p_{11} \sigma_{11} \sigma_{12}-p_{12}^{-1} \sigma_{21} \sigma_{12}+\sigma_{11} \sigma_{12}$. If $A$ is a noetherian AS-regular algebra with Nakayama automorphism $\mu_{A}$ generated in degree 1, the associated trimmed double Ore extension $A_{P}\left[y_{1}, y_{2} ; \sigma\right]$ is noetherian and $\sigma$ is invertible, by Theorem 4.3. we have Nakayama automorphism $\mu$ of $A_{P}\left[y_{1}, y_{2} ; \sigma, \delta\right]$ satisfying

$$
\begin{aligned}
& \mu(a)=(\operatorname{det} \sigma)^{-1} \circ \mu_{A}(a), \quad \forall a \in A \\
& \mu\left(\begin{array}{l}
y_{1} \\
y_{2}
\end{array}\right)=\operatorname{hdet} \sigma \cdot\left(\begin{array}{cc}
p_{12}^{-1} & 0 \\
p_{11}\left(1+p_{12}^{-1}\right) & p_{12}
\end{array}\right)\left(\begin{array}{l}
y_{1} \\
y_{2}
\end{array}\right)+\left(\begin{array}{l}
a_{1} \\
a_{2}
\end{array}\right),
\end{aligned}
$$

for some $a_{1}, a_{2} \in A$. In particular, if $A$ is Koszul and $\delta=0$, this Nakayama automorphism of trimmed double Ore extension $A_{P}\left[y_{1}, y_{2} ; \sigma\right]$ is the same to the one obtained in [21, Theorem 2] by Remark 4.4

Example 5.3. Let $k$ be a field of characteristic 0 . We consider the Nakayama automorphism of the example constructed in [18, Example 4.3]. Let $A=k\left\langle x_{1}, x_{2}\right\rangle /\left(f_{1}, f_{2}\right)$, where $f_{1}=x_{1}^{2} x_{2}-x_{2} x_{1}^{2}$ and $f_{2}=x_{1} x_{2}^{2}-x_{2}^{2} x_{1}$. In fact, $A$ is a noetherian 3-Koszul AS-regular algebra, and the Nakayama automorphism $\mu_{A}$ of $A$ is

$$
\mu_{A}\left(x_{1}\right)=-x_{1}, \quad \mu_{A}\left(x_{2}\right)=-x_{2} .
$$

Define a graded normal linear map $\tau=(\sigma, 0)$ from $A \otimes A$ to $A \otimes A$ by

$$
\begin{aligned}
& \tau\left(x_{1} \otimes x_{1}\right)=p x_{2} \otimes x_{1}+p x_{2} \otimes x_{2}, \\
& \tau\left(x_{1} \otimes x_{2}\right)=p x_{1} \otimes x_{1}+p x_{1} \otimes x_{2}, \\
& \tau\left(x_{2} \otimes x_{1}\right)=p x_{2} \otimes x_{1}-p x_{2} \otimes x_{2}, \\
& \tau\left(x_{2} \otimes x_{2}\right)=p x_{1} \otimes x_{1}-p x_{1} \otimes x_{2},
\end{aligned}
$$

where $p \in k^{\times}$. The algebra homomorphism $\sigma: A \rightarrow \mathbb{M}_{2}(A)$ is invertible with inverse $\varphi$ satisfying:

$$
\sigma\left(x_{1}\right)=p\left(\begin{array}{cc}
x_{2} & x_{2} \\
x_{2} & -x_{2}
\end{array}\right), \quad \sigma\left(x_{2}\right)=p\left(\begin{array}{cc}
x_{1} & x_{1} \\
x_{1} & -x_{1}
\end{array}\right) .
$$


The twisted tensor product $A \otimes^{\tau} A$ is isomorphic to the algebra $C:=k\left\langle x_{1}, x_{2}, y_{1}, y_{2}\right\rangle /(G)$, where $G=\left\{g_{i}\right\}_{i=1}^{8}$, and

$$
\begin{array}{ll}
g_{1}=x_{1}^{2} x_{2}-x_{2} x_{1}^{2}, & g_{2}=x_{1} x_{2}^{2}-x_{2}^{2} x_{1}, \\
g_{3}=y_{1}^{2} y_{2}-y_{2} y_{1}^{2}, & g_{4}=y_{1} y_{2}^{2}-y_{2}^{2} y_{1}, \\
g_{5}=y_{1} x_{1}-p x_{2} y_{1}-p x_{2} y_{2}, & g_{6}=y_{1} x_{2}-p x_{1} y_{1}-p x_{1} y_{2}, \\
g_{7}=y_{2} x_{1}-p x_{2} y_{1}+p x_{2} y_{2}, & g_{8}=y_{2} x_{2}-p x_{1} y_{1}+p x_{1} y_{2} .
\end{array}
$$

The algebra $C$ is also a noetherian AS-regular algebra by Theorem 2.11. We compute the Nakayama automorphism of $C$ in the following.

A minimal free resolution of ${ }_{A} k$ is

$$
P^{*}: 0 \rightarrow A(-4) \stackrel{d^{-3}}{\longrightarrow} A(-3)^{\oplus 2} \stackrel{d^{-2}}{\longrightarrow} A(-1)^{\oplus 2} \stackrel{d^{-1}}{\longrightarrow} A \rightarrow{ }_{A} k \rightarrow 0,
$$

where

$$
d^{-1}=\left(\begin{array}{c}
x_{1} \\
x_{2}
\end{array}\right), \quad d^{-2}=\left(\begin{array}{cc}
-x_{2} x_{1} & x_{1}^{2} \\
-x_{2}^{2} & x_{1} x_{2}
\end{array}\right), \quad d^{-3}=\left(\begin{array}{ll}
-x_{2} & x_{1}
\end{array}\right) .
$$

Firstly, we calculate the determinant of $\sigma$. We construct two algebra homomorphisms $\phi_{2}: A \rightarrow \mathbb{M}_{2}(A)$ and $\operatorname{det} \sigma: A \rightarrow A$ to make complex $A \otimes^{\tau} P$. exact in $\operatorname{Gr}\left(C \otimes A^{o}\right)$ as in Theorem 2.9, that is,

$$
0 \rightarrow C(-4)^{\operatorname{det} \sigma} \rightarrow\left(C(-3)^{\oplus 2}\right)^{\phi_{2}} \rightarrow\left(C(-1)^{\oplus 2}\right)^{\sigma} \rightarrow C \rightarrow A \rightarrow 0,
$$

where

$$
\begin{array}{ll}
\phi_{2}\left(x_{1}\right)=-2 p^{3}\left(\begin{array}{cc}
x_{2} & x_{2} \\
x_{2} & -x_{2}
\end{array}\right), & \phi_{2}\left(x_{2}\right)=-2 p^{3}\left(\begin{array}{cc}
x_{1} & x_{1} \\
x_{1} & -x_{1}
\end{array}\right), \\
\operatorname{det} \sigma\left(x_{1}\right)=4 p^{4} x_{1}, & \operatorname{det} \sigma\left(x_{2}\right)=4 p^{4} x_{2} .
\end{array}
$$

Now we turn to compute the homological determinant of $\sigma$. We have the following commutative diagram

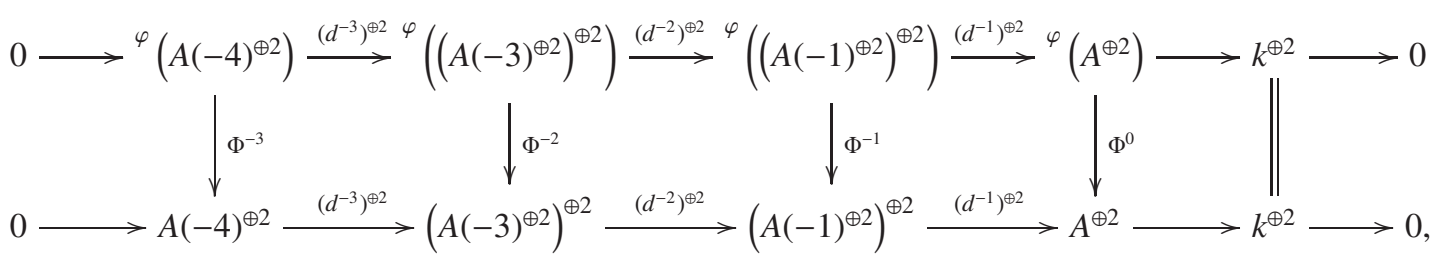

where

$\Phi^{0}\left(\begin{array}{l}a_{1} \\ a_{2}\end{array}\right)=\left(\begin{array}{l}\sigma_{11}\left(a_{1}\right)+\sigma_{21}\left(a_{2}\right) \\ \sigma_{12}\left(a_{1}\right)+\sigma_{22}\left(a_{2}\right)\end{array}\right)$,

$\Phi^{-1}\left(\begin{array}{l}\left(a_{1}, a_{2}\right) \\ \left(b_{1}, b_{2}\right)\end{array}\right)=p\left(\begin{array}{l}\left(\sigma_{11}\left(a_{2}\right)+\sigma_{12}\left(a_{2}\right)+\sigma_{21}\left(b_{2}\right)+\sigma_{22}\left(b_{2}\right), \sigma_{11}\left(a_{1}\right)+\sigma_{12}\left(a_{1}\right)+\sigma_{21}\left(b_{1}\right)+\sigma_{22}\left(b_{1}\right)\right) \\ \left(\sigma_{11}\left(a_{2}\right)-\sigma_{12}\left(a_{2}\right)+\sigma_{21}\left(b_{2}\right)-\sigma_{22}\left(b_{2}\right), \sigma_{11}\left(a_{1}\right)-\sigma_{12}\left(a_{1}\right)+\sigma_{21}\left(b_{1}\right)-\sigma_{22}\left(b_{1}\right)\right)\end{array}\right)$,

$\Phi^{-2}\left(\begin{array}{l}\left(a_{1}, a_{2}\right) \\ \left(b_{1}, b_{2}\right)\end{array}\right)=-2 p^{3}\left(\begin{array}{l}\left.\left(\sigma_{11}\left(a_{2}\right)+\sigma_{12}\left(a_{2}\right)+\sigma_{21}\left(b_{2}\right)+\sigma_{22}\left(b_{2}\right), \sigma_{11}\left(a_{1}\right)+\sigma_{12}\left(a_{1}\right)+\sigma_{21}\left(b_{1}\right)+\sigma_{22}\left(b_{1}\right)\right)\right) \\ \left(\sigma_{11}\left(a_{2}\right)-\sigma_{12}\left(a_{2}\right)+\sigma_{21}\left(b_{2}\right)-\sigma_{22}\left(b_{2}\right), \sigma_{11}\left(a_{1}\right)-\sigma_{12}\left(a_{1}\right)+\sigma_{21}\left(b_{1}\right)-\sigma_{22}\left(b_{1}\right)\right)\end{array}\right)$,

$\Phi^{-3}\left(\begin{array}{l}a_{1} \\ a_{2}\end{array}\right)=4 p^{4}\left(\begin{array}{l}\sigma_{11}\left(a_{1}\right)+\sigma_{21}\left(a_{2}\right) \\ \sigma_{12}\left(a_{1}\right)+\sigma_{22}\left(a_{2}\right)\end{array}\right)$,

for $a_{1}, a_{2}, b_{1}, b_{2} \in A$. By the definition, we have

$$
\operatorname{hdet} \sigma=\left(\begin{array}{cc}
4 p^{4} & 0 \\
0 & 4 p^{4}
\end{array}\right) .
$$


By Theorem 4.3 , we have the Nakayama automorphism $\mu_{C}$ satisfying

$$
\begin{array}{ll}
\mu_{C}\left(x_{1}\right)=-\left(4 p^{4}\right)^{-1} x_{1}, & \mu_{C}\left(x_{2}\right)=-\left(4 p^{4}\right)^{-1} x_{2}, \\
\mu_{C}\left(y_{1}\right)=-4 p^{4} y_{1}, & \mu_{C}\left(y_{2}\right)=-4 p^{4} y_{2} .
\end{array}
$$

Acknowledgments. Y. Shen is supported by NSFC (Grant No.11626215) and Science Foundation of Zhejiang Sci-Tech University (ZSTU) under Grant No.16062066-Y. G.-S. Zhou is supported by NSFC (Grant No. 11601480). D.-M. Lu is supported by NSFC (Grant No. 11671351).

\section{REFERENCES}

[1] M. Artin and W. Schelter, Graded algebras of global dimension 3, Adv. Math., 66(2) (1987), 171-216.

[2] R. Berger and N. Marconnet, Koszul and Gorenstein properties for homogeneous algebras, Algebr. Represent. Theory, 9(1) (2006), 67-97.

[3] S. Caenepeel, B. Ion, G. Militaru and S. Zhu, The factorization problem and the smash biproduct of algebras and coalgebras, Algebr. Represent. Theory, 3(1) (2000), 19-42.

[4] A. Čap, H. Schichl and J. Vanžura, On twisted tensor products of algebras, Comm. Algebra, 23(12) (1995), 4701-4735.

[5] K. Chan, C Walton and J. J. Zhang, Hopf actions and Nakayama automorphisms, J. Alg., 409 (2014), $26-53$.

[6] P. Jøgensen and J. J. Zhang, Gourmet's guide to gorensteiness, Adv. Math., 151 (2000), 313-345.

[7] L.-Y. Liu, S.-Q. Wang and Q.-S. Wu, Twisted Calabi-Yau property of Ore extensions, J. Noncommut. Geom., 7 (2014), 587-609.

[8] D.-M. Lu, J. H. Palmieri, Q.-S. Wu and J. J. Zhang, Kosuzl equivalences in A $\infty_{\infty}$-algebras, New York J. Math., 14 (2008), $325-378$.

[9] J.-F. Lü, X.-F. Mao and J. J. Zhang, Nakayama automorphism and applications, Trans. Amer. Math. Soc., 369 (2017), 24252460.

[10] M. Reyes, D. Rogalski, and J. J. Zhang, Skew Calabi-Yau algebras and homological identities, Adv. Math.,264 (2014), $308-354$.

[11] M. Reyes, D. Rogalski, and J. J. Zhang, Skew Calabi-Yau triangulated categories and Frobenius Ext-algebras., to appear in Trans. Amer. Math. Soc..

[12] Y. Shen, X. Wang and G.-S. Zhou, Ext-algebras of graded skew extensions and $A_{\infty}$-structures, arXiv:1707.01610 2017.

[13] S. P. Smith, Some finite dimensional algebras related to elliptic curves, CMS Conf. Proc., 19 (1996), $315-348$.

[14] D. R. Stephenson and J. J. Zhang, Growth of graded Noetherian rings, Proc. Amer. Math. Soc., 125(6) (1997), 1593-1605.

[15] A. Van Daele and S. Van Keer, The Yang-Baxter and pentagon equation, Compositio Math., 91(2) (1994), $201-221$.

[16] M. Van den Bergh, A relation between Hochschild homology and cohomology for Gorenstein rings, Proc. Amer. Math. Soc., 126 (1998), 1345-1348.

[17] C. Walton and S. Witherspoon, PBW deformations of braided products, arXiv:1601.02274 2, 2017.

[18] X. Wang, Y. Shen and D.-M. Lu, Artin-Schelter regularity of R-smash products, preprint.

[19] A. Yekutieli and J. J. Zhang, Homological transcendence degree, Proc. Lond. Math. Soc., 93(3) (2006), $105-137$.

[20] J. J. Zhang and J. Zhang, Double Ore extension, J. Pure Appl. Algebra, 212(12) (2008), 2668-2690.

[21] C. Zhu, F. Van Oystaeyen and Y.-H. Zhang, Nakayama automorphisms of double Ore extensions of Koszul regular algebras, Mauscripta Math., 152 (2017), 555-584.

Shen: Department of Mathematics, Zhejiang Sci-Tech University, Hangzhou 310018, China

E-mail address: yuanshen@zstu.edu.cn

Zhou: Ningbo Institute of Technology, Zhejang University, Ningbo 315100, China

E-mail address: $10906045 @ z j u . e d u . c n$

Lu: School of Mathematical Sciences, Zhejiang University, Hangzhou 310027, China

E-mail address: dmlu@zju.edu.cn 